\title{
NON-EQUILIBRIUM DYNAMICS OF A THERMAL PLASMA IN A GRAVITATIONAL FIELD
}

\author{
Antonio Campos \\ Department of Physics, University of Maryland, College Park, Maryland 20742; \\ Institut de Física d'Altes Energies, Universitat Autònoma de Barcelona, 08193 Bellaterra (Barcelona), Spain; \\ Center for Theoretical Physics, Laboratory for Nuclear Science, Massachusetts Institute of Technology, Cambridge, \\ Massachusetts 02139* \\ B. L. Hu \\ Department of Physics, University of Maryland, College Park, Maryland 20742
}

Report umdpp \#98-98/MIT-CTP 2745

(May 27, 2022)

\begin{abstract}
We introduce functional methods to study the non-equilibrium dynamics of a quantum massless scalar field at finite temperature in a gravitational field. We calculate the close time path (CTP) effective action and, using its formal equivalence with the influence functional, derive the noise and dissipation kernels of the quantum open system in terms of quantities in thermodynamical equilibrium. Using this fact, we formally prove the existence of a fluctuation-dissipation relation (FDR) at all temperatures between the quantum fluctuations of the plasma in thermal equilibrium and the energy dissipated by the external gravitational field. What is new is the identification of a stochastic source (noise) term arising from the quantum and thermal fluctuations in the plasma field, and the derivation of a Langevin-type equation which describes the non-equilibrium dynamics of the gravitational field influenced by the plasma. The back reaction of the plasma on the gravitational field is embodied in the FDR. From the CTP effective action the contribution of the quantum scalar field to the thermal graviton polarization tensor can also be derived and it is shown to agree with other techniques, most notably, linear response theory (LRT). We show the connection between the LRT, which is applicable for near-equilibrium conditions and the functional methods used in this work which are useful for fully non-equilibrium conditions.
\end{abstract}

PACS number(s): 11.10.Wx, 05.40.+j, $-04.62 .+\mathrm{v}$

\section{INTRODUCTION}

The behavior of a relativistic quantum field at finite temperature in a gravitational field has been studied before by Gross, Perry and Yaffe [1], Gribosky, Donoghue and Holstein [2], Rebhan and co-workers [3], de Almeida, Brandt, Frenkel and Taylor [4,5] for scalar and Abelian gauge fields. The thermal graviton polarization tensor and the effective action have been calculated and applied to the study of stability of hot flat/curved spaces, and dynamics of cosmological perturbations. Using linear response theory (LRT) [7] 12], Jackiw and Nair [6] derived the response function at high temperatures for a non-Abelian gauge field in a thermal plasma of charged particles.

To describe screening effects and stability of thermal quantum gravity, one needs only the real part of the polarization tensor; but for damping effects, the imaginary part is essential. The gravitational polarization tensor obtained from the thermal graviton self-energy represents only a part (the thermal correction to the vacuum polarization) of the finite temperature quantum stress tensor, which under more general conditions (e. g. dynamical background) should contain also contributions from particle creation (arising from vacuum fluctuations at zero and finite temperature). These processes engender dissipation in the dynamics of the gravitational field and their fluctuations appear as noise in the plasma. These latter two effects have not been sufficiently appreciated in most previous works in thermal field theory.

*Present address 
In this work we use open system concepts and functional methods a la Schwinger-Keldysh [13, 14, and FeynmanVernon [15]. This framework encompasses LRT which we illustrate in a calculation of the quantum corrections of the scalar field to the thermal graviton polarization tensor with results identical to that obtained before [3, 4]. More importantly, we derive the noise and dissipation kernels, prove that they satisfy a fluctuation-dissipation relation (FDR) 99,10,12] at all temperatures, and derive a stochastic semiclassical equation for the non-equilibrium dynamics of the gravitational field under the influence of the thermal plasma including all three aspects mentioned above. These cannot be obtained easily by LRT and other thermal field theory methods.

Here the thermal plasma will be described by a massless scalar quantum field at finite temperature and the gravitational field by a linear perturbation from Minkowski spacetime. Since the plasma is considered to be in thermal equilibrium it will be characterized by a time-like normalized vector field $u^{\mu}$, representing the four-velocity of the plasma, and the temperature of the fluid in its own rest frame $\beta^{-1}$. Taking into account the four-velocity $u^{\mu}$ of the plasma, a manifestly Lorentz-covariant approach to thermal field theory may be used [16]. However, in order to simplify the involved tensorial structure we work in the co-moving coordinate system of the plasma where $u^{\mu}=(1,0,0,0)$.

By making conformal transformations on the field and the spacetime, our results may be easily generalized to the case of a conformally coupled quantum scalar field at finite temperature in a spatially flat Friedmann-Robertson-Walker universe 17]. Indeed we have earlier used the functional method and the Brownian motion paradigm [13, 15, 18, 19 to study similar problems in semiclassical gravity [20]. We found that quantum noise arising from fluctuations in the particle creation would constitute a stochastic source hitherto undiscovered [21] (whose effect can overdominate the expectation value of the energy momentum tensor in the semiclassical Einstein equation 22 24) in a new form of Einstein-Langevin equation 25 33]. We also came to the understanding that back reaction of vacuum quantum field processes (such as particle creation) on the dynamics of the early universe near the Planck time is summarily a manifestation of a FDR in semiclassical gravity.

The close time path (CTP) functional formalism of Schwinger [13] is based on a generating functional defined in such a way that expectation values (rather than matrix elements) of time and anti-time ordered field products are directly computed. Keldysh [14] developed a diagramatic technique approach useful for non-equilibrium processes in quantum field theory. This method has been further developed in [34 36] (For some applications in field theory and cosmology see 37 41,25,42 46]). The advantage of this formalism is that effective dynamical equations for the expectation value of the quantum field may be derived from the effective action. Since these effective equations are real and causal a proper initial value problem may be formulated. The generalization of this formalism to curved spacetimes was given by DeWitt and Jordan, Calzetta and $\mathrm{Hu}$ 47, 48, 37.

In Sec. II], we describe our model and the derivation of the thermal CTP effective action. From this effective action we identify the dissipation and the noise kernels representing the linear response and the quantum fluctuations of the plasma, respectively, and obtain a stochastic semiclassical equation for the gravitational perturbations. This is done by using the underlying ideas of the influence functional formalism of Feynman and Vernon [15]. Sec. [II] is devoted to showing the existence of the FDR. We formally show that the noise and dissipation kernels extracted from the CTP effective action in general satisfy a formal relation at all temperatures. By explicitly computing the corresponding kernels we show that this relation is satisfied at high and zero temperature. In Sec. IV we describe how our functional approach reproduces results from LRT. Finally, in Sec. V, we give a brief conclusion. To make this presentation self-contained, in Appendix A we give a brief yet complete description of thermal field theory via the CTP functional formalism.

\section{CTP EFFECTIVE ACTION AT FINITE TEMPERATURE}




\section{A. The model}

In this section, we derive the CTP effective action for a thermal plasma in a gravitational field. To describe the plasma we consider a free massless scalar field $\phi$ arbitrarily coupled to a gravitational field $g_{\mu \nu}$ with classical action

$$
S_{m}\left[\phi, g_{\mu \nu}\right]=-\frac{1}{2} \int d^{n} x \sqrt{-g}\left[g^{\mu \nu} \partial_{\mu} \phi \partial_{\nu} \phi+\xi(n) R \phi^{2}\right]
$$

where $R(x)$ is the scalar curvature and the arbitrary parameter $\xi(n)$ defines the type of coupling between the scalar field and the gravitational field. If $\xi(n)=0$ the quantum field is said to be minimally coupled, if $\xi(n)=(n-2) /[4(n-1)]$, where $n$ is the spacetime dimensions, the field is said to be conformally coupled. To describe the gravitational field we consider a small deviation from flat spacetime

$$
g_{\mu \nu}(x)=\eta_{\mu \nu}+h_{\mu \nu}(x)
$$

with signature $(-,+, \cdots,+)$ for the Minkowski metric. Using this metric and neglecting the surface terms that appear in an integration by parts, the action for the scalar field may be written perturbatively as

$$
S_{m}\left[\phi, h_{\mu \nu}\right]=\frac{1}{2} \int d^{n} x \phi\left[\square+V^{(1)}+V^{(2)}+\cdots\right] \phi,
$$

where the first and second order perturbative operators $V^{(1)}$ and $V^{(2)}$ are given by

$$
\begin{aligned}
V^{(1)} & \equiv-\left\{\left[\partial_{\mu} \bar{h}^{\mu \nu}(x)\right] \partial_{\nu}+\bar{h}^{\mu \nu}(x) \partial_{\mu} \partial_{\nu}+\xi(n) R^{(1)}(x)\right\}, \\
V^{(2)} & \equiv\left\{\left[\partial_{\mu} \hat{h}^{\mu \nu}(x)\right] \partial_{\nu}+\hat{h}^{\mu \nu}(x) \partial_{\mu} \partial_{\nu}-\xi(n)\left(R^{(2)}(x)+\frac{1}{2} h(x) R^{(1)}(x)\right)\right\} .
\end{aligned}
$$

In the above expressions, $R^{(k)}$ is the $k$-order term in the pertubation $h_{\mu \nu}(x)$ of the scalar curvature and the definitions $\bar{h}_{\mu \nu}$ and $\hat{h}_{\mu \nu}$ denote a linear and a quadratic combination of the perturbation, respectively,

$$
\begin{aligned}
& \bar{h}_{\mu \nu} \equiv h_{\mu \nu}-\frac{1}{2} h \eta_{\mu \nu} \\
& \hat{h}_{\mu \nu} \equiv h_{\mu}^{\alpha} h_{\alpha \nu}-\frac{1}{2} h h_{\mu \nu}+\frac{1}{8} h^{2} \eta_{\mu \nu}-\frac{1}{4} h_{\alpha \beta} h^{\alpha \beta} \eta_{\mu \nu} .
\end{aligned}
$$

For the gravitational field we take the following action

$$
\begin{aligned}
S_{g}^{d i v}\left[g_{\mu \nu}\right]= & \frac{1}{\ell_{P}^{n-2}} \int d^{n} x \sqrt{-g} R(x) \\
& +\frac{\alpha \bar{\mu}^{n-4}}{4(n-4)} \int d^{n} x \sqrt{-g}\left[3 R_{\mu \nu \alpha \beta}(x) R^{\mu \nu \alpha \beta}(x)-\left(1-360\left(\xi(n)-\frac{1}{6}\right)^{2}\right) R(x) R(x)\right] .
\end{aligned}
$$

The first term is the classical Einstein-Hilbert action and the second divergent term in four dimensions is the counterterm used in order to renormalize the effective action. In this action $\ell_{P}^{2}=16 \pi G, \alpha=\left(2880 \pi^{2}\right)^{-1}$ and $\bar{\mu}$ is an arbitrary mass scale. It is noteworthy that the counterterms are independent of the temperature because the thermal contribution to the effective action is finite and does not include additional divergencies.

\section{B. CTP effective action}

In Appendix A we described how to compute the CTP effective action at finite temperature for a quantum scalar free field theory. In fact, we need to generalize the CTP formalism for two fields in the semiclassical approximation. This generalization, which is justified in Appendix A3, gives the following formal effective action (replace $\phi$ by $h_{\mu \nu}$ and $\psi$ by $\phi$ in equation (A24)) 


$$
\Gamma_{C T P}^{\beta}\left[h_{\mu \nu}^{ \pm}\right]=S_{g}^{d i v}\left[h_{\mu \nu}^{+}\right]-S_{g}^{d i v}\left[h_{\mu \nu}^{-}\right]-\frac{i}{2} \operatorname{Tr}\left\{\ln \bar{G}_{a b}^{\beta}\left[h_{\mu \nu}^{ \pm}\right]\right\},
$$

where \pm denote the forward and backward time path of the CTP formalism and $\bar{G}_{a b}^{\beta}\left[h_{\mu \nu}^{ \pm}\right]$is the complete $2 \times 2$ matrix propagator ( $a$ and $b$ take \pm values) with thermal boundary conditions for the differential operator $\square+V^{(1)}+V^{(2)}+\cdots$. In fact, the actual form of $\bar{G}_{a b}^{\beta}$ cannot be explicitly given. However, it is easy to obtain a perturbative expansion in terms of $V_{a b}^{(k)}$, the $k$-order matrix version of the complete differential operator defined by $V_{ \pm \pm}^{(k)} \equiv \pm V_{ \pm}^{(k)}$ and $V_{ \pm \mp}^{(k)} \equiv 0$, and $G_{a b}^{\beta}$, the thermal matrix propagator for a massless scalar field in flat spacetime (A15 A16). To second order $\bar{G}_{a b}^{\beta}$ reads,

$$
\bar{G}_{a b}^{\beta}=G_{a b}^{\beta}-G_{a c}^{\beta} V_{c d}^{(1)} G_{d b}^{\beta}-G_{a c}^{\beta} V_{c d}^{(2)} G_{d b}^{\beta}+G_{a c}^{\beta} V_{c d}^{(1)} G_{d e}^{\beta} V_{e f}^{(1)} G_{f b}^{\beta}+\cdots
$$

Expanding the logarithm and dropping one term independent of the perturbation $h_{\mu \nu}^{ \pm}(x)$, the CTP effective action may be perturbatively written as,

$$
\begin{aligned}
\Gamma_{C T P}^{\beta}\left[h_{\mu \nu}^{ \pm}\right]= & S_{g}^{d i v}\left[h_{\mu \nu}^{+}\right]-S_{g}^{d i v}\left[h_{\mu \nu}^{-}\right] \\
& +\frac{i}{2} \operatorname{Tr}\left[V_{+}^{(1)} G_{++}^{\beta}-V_{-}^{(1)} G_{--}^{\beta}+V_{+}^{(2)} G_{++}^{\beta}-V_{-}^{(2)} G_{--}^{\beta}\right] \\
& -\frac{i}{4} \operatorname{Tr}\left[V_{+}^{(1)} G_{++}^{\beta} V_{+}^{(1)} G_{++}^{\beta}+V_{-}^{(1)} G_{--}^{\beta} V_{-}^{(1)} G_{--}^{\beta}-2 V_{+}^{(1)} G_{+-}^{\beta} V_{-}^{(1)} G_{-+}^{\beta}\right] .
\end{aligned}
$$

The next task is to identify the noise and dissipation kernels from the non-local terms of the above thermal CTP effective action. The last trace is the only term that is responsible for dissipation because all others are constructed with symmetric kernels. In fact, this non-local term have information of the noise kernel also, as we will see in the next section when we show the existence of a FDR. In order to identify the role of each kernel we have to compute all the traces in the formal expression for the effective action. Some of these traces have divergencies that are canceled using the counterterms introduced in the classical gravitational action after dimensional regularization. In general, the non-local pieces are of the form $\operatorname{Tr}\left[V_{a}^{(1)} G_{m n}^{\beta} V_{b}^{(1)} G_{r s}^{\beta}\right]$. In terms of the Fourier transformed thermal propagators $\tilde{G}_{a b}^{\beta}(k)$ these traces can be written as,

$$
\operatorname{Tr}\left[V_{a}^{(1)} G_{m n}^{\beta} V_{b}^{(1)} G_{r s}^{\beta}\right]=\int d^{n} x d^{n} x^{\prime} h_{\mu \nu}^{a}(x) h_{\alpha \beta}^{b}\left(x^{\prime}\right) \int \frac{d^{n} k}{(2 \pi)^{n}} \frac{d^{n} q}{(2 \pi)^{n}} e^{i k \cdot\left(x-x^{\prime}\right)} \tilde{G}_{m n}^{\beta}(k+q) \tilde{G}_{r s}^{\beta}(q) \mathrm{T}^{\mu \nu, \alpha \beta}(q, k),
$$

where the tensor $\mathrm{T}^{\mu \nu, \alpha \beta}(q, k)$ is defined in Appendix B. In particular, the last trace of (9) may be split in two different kernels $\mathrm{N}^{\mu \nu, \alpha \beta}\left(x-x^{\prime}\right)$ and $\mathrm{D}^{\mu \nu, \alpha \beta}\left(x-x^{\prime}\right)$,

$$
\frac{i}{2} \operatorname{Tr}\left[V_{+}^{(1)} G_{+-}^{\beta} V_{-}^{(1)} G_{-+}^{\beta}\right]=-\int d^{4} x d^{4} x^{\prime} h_{\mu \nu}^{+}(x) h_{\alpha \beta}^{-}\left(x^{\prime}\right)\left[\mathrm{D}^{\mu \nu, \alpha \beta}\left(x-x^{\prime}\right)+i \mathrm{~N}^{\mu \nu, \alpha \beta}\left(x-x^{\prime}\right)\right] .
$$

Recalling Eq. A16 one can express the Fourier transforms of these kernels, respectively, as

$$
\begin{aligned}
& \tilde{\mathrm{N}}^{\mu \nu, \alpha \beta}(k)=\pi^{2} \int \frac{d^{4} q}{(2 \pi)^{4}}\left\{\theta\left(k^{o}+q^{o}\right) \theta\left(-q^{o}\right)+\theta\left(-k^{o}-q^{o}\right) \theta\left(q^{o}\right)+n_{\beta}\left(\left|q^{o}\right|\right)+n_{\beta}\left(\left|k^{o}+q^{o}\right|\right)\right. \\
& \left.+2 n_{\beta}\left(\left|q^{o}\right|\right) n_{\beta}\left(\left|k^{o}+q^{o}\right|\right)\right\} \delta\left(q^{2}\right) \delta\left[(k+q)^{2}\right] \mathrm{T}^{\mu \nu, \alpha \beta}(q, k), \\
& \tilde{\mathrm{D}}^{\mu \nu, \alpha \beta}(k)=-i \pi^{2} \int \frac{d^{4} q}{(2 \pi)^{4}}\left\{\theta\left(k^{o}+q^{o}\right) \theta\left(-q^{o}\right)-\theta\left(-k^{o}-q^{o}\right) \theta\left(q^{o}\right)+s g\left(k^{o}+q^{o}\right) n_{\beta}\left(\left|q^{o}\right|\right)\right. \\
& \left.-s g\left(q^{o}\right) n_{\beta}\left(\left|k^{o}+q^{o}\right|\right)\right\} \delta\left(q^{2}\right) \delta\left[(k+q)^{2}\right] \mathrm{T}^{\mu \nu, \alpha \beta}(q, k) .
\end{aligned}
$$

Using the property $\mathrm{T}^{\mu \nu, \alpha \beta}(q, k)=\mathrm{T}^{\mu \nu, \alpha \beta}(-q,-k)$, it is easy to see that $\mathrm{N}^{\mu \nu, \alpha \beta}\left(x-x^{\prime}\right)$ is symmetric and $\mathrm{D}^{\mu \nu, \alpha \beta}\left(x-x^{\prime}\right)$ antisymmetric in their arguments; that is, $\mathrm{N}^{\mu \nu, \alpha \beta}(x)=\mathrm{N}^{\mu \nu, \alpha \beta}(-x)$ and $\mathrm{D}^{\mu \nu, \alpha \beta}(x)=-\mathrm{D}^{\mu \nu, \alpha \beta}(-x)$. To properly identify these kernels with the noise and the dissipation of the system, respectively, we have to write the renormalized 
CTP effective action at finite temperature (9) in an influence functional form 15. The imaginary part of the CTP effective action written in this form can be identified with the noise kernel and the antisymmetric piece of the real part with the dissipation kernel. This identification will be finally justified in two ways. In Sec. III we will see that these kernels satisfy a thermal FDR and in Sec. [V] we will see from the point of view of the LRT that $\mathrm{D}^{\mu \nu, \alpha \beta}(x)$ corresponds to the dissipation of the gravitational field or equivalently to the response of the thermal plasma to the gravitational perturbation, and $\mathrm{N}^{\mu \nu, \alpha \beta}(x)$ to the random fluctuations of the plasma.

If we denote the difference and the sum of the perturbations $h_{\mu \nu}^{ \pm}$defined along each branch $C_{ \pm}$of the complex time path of integration $C$ by $\left[h_{\mu \nu}\right] \equiv h_{\mu \nu}^{+}-h_{\mu \nu}^{-}$and $\left\{h_{\mu \nu}\right\} \equiv h_{\mu \nu}^{+}+h_{\mu \nu}^{-}$, respectively, the influence functional form of the thermal CTP effective action may be written to second order in $h_{\mu \nu}$ as,

$$
\begin{aligned}
\Gamma_{C T P}^{\beta}\left[h_{\mu \nu}^{ \pm}\right] \simeq & \frac{1}{2 \ell_{P}^{2}} \int d^{4} x d^{4} x^{\prime}\left[h_{\mu \nu}\right](x) \mathrm{L}_{(o)}^{\mu \nu, \alpha \beta}\left(x-x^{\prime}\right)\left\{h_{\alpha \beta}\right\}\left(x^{\prime}\right) \\
& +\frac{1}{2} \int d^{4} x\left[h_{\mu \nu}\right](x) T_{(\beta)}^{\mu \nu} \\
& +\frac{1}{2} \int d^{4} x d^{4} x^{\prime}\left[h_{\mu \nu}\right](x) \mathrm{H}^{\mu \nu, \alpha \beta}\left(x-x^{\prime}\right)\left\{h_{\alpha \beta}\right\}\left(x^{\prime}\right) \\
& -\frac{1}{2} \int d^{4} x d^{4} x^{\prime}\left[h_{\mu \nu}\right](x) \mathrm{D}^{\mu \nu, \alpha \beta}\left(x-x^{\prime}\right)\left\{h_{\alpha \beta}\right\}\left(x^{\prime}\right) \\
& +\frac{i}{2} \int d^{4} x d^{4} x^{\prime}\left[h_{\mu \nu}\right](x) \mathrm{N}^{\mu \nu, \alpha \beta}\left(x-x^{\prime}\right)\left[h_{\alpha \beta}\right]\left(x^{\prime}\right) .
\end{aligned}
$$

The first line is the Einstein-Hilbert action to second order in the perturbation $h_{\mu \nu}^{ \pm}(x) . \mathrm{L}_{(o)}^{\mu \nu, \alpha \beta}(x)$ is a symmetric kernel (i.e. $\left.\mathrm{L}_{(o)}^{\mu \nu, \alpha \beta}(x)=\mathrm{L}_{(o)}^{\mu \nu, \alpha \beta}(-x)\right)$ and its Fourier transform is given by

$$
\tilde{\mathrm{L}}_{(o)}^{\mu \nu, \alpha \beta}(k)=\frac{1}{4}\left[-k^{2} \mathrm{~T}_{1}^{\mu \nu, \alpha \beta}(q, k)+2 k^{2} \mathrm{~T}_{4}^{\mu \nu, \alpha \beta}(q, k)+\mathrm{T}_{8}^{\mu \nu, \alpha \beta}(q, k)-2 \mathrm{~T}_{13}^{\mu \nu, \alpha \beta}(q, k)\right] .
$$

The fourteen elements of the tensor basis $\mathrm{T}_{i}^{\mu \nu, \alpha \beta}(q, k)(i=1, \cdots, 14)$ are defined in Appendix $\mathrm{B}$. In the second line $T_{(\beta)}^{\mu \nu}$ has the form of a perfect fluid stress-energy tensor

$$
T_{(\beta)}^{\mu \nu}=\frac{\pi^{2}}{30 \beta^{4}}\left[u^{\mu} u^{\nu}+\frac{1}{3}\left(\eta^{\mu \nu}+u^{\mu} u^{\nu}\right)\right],
$$

where $u^{\mu}$ is the four-velocity of the plasma and the factor $\frac{\pi^{2}}{30 \beta^{4}}$ is the familiar thermal energy density for massless scalar particles at temperature $\beta^{-1}$. In the third line, the Fourier transform of the symmetric kernel $\mathrm{H}^{\mu \nu, \alpha \beta}(x)$ can be expressed as

$$
\begin{aligned}
\tilde{\mathrm{H}}^{\mu \nu, \alpha \beta}(k)= & -\frac{\alpha k^{4}}{4}\left\{\frac{1}{2} \ln \frac{\left|k^{2}\right|}{\mu^{2}} \mathrm{Q}^{\mu \nu, \alpha \beta}(k)+\frac{1}{3} \overline{\mathrm{Q}}^{\mu \nu, \alpha \beta}(k)\right\} \\
& +\frac{\pi^{2}}{180 \beta^{4}}\left\{-\mathrm{T}_{1}^{\mu \nu, \alpha \beta}(u, k)-2 \mathrm{~T}_{2}^{\mu \nu, \alpha \beta}(u, k)+\mathrm{T}_{4}^{\mu \nu, \alpha \beta}(u, k)+2 \mathrm{~T}_{5}^{\mu \nu, \alpha \beta}(u, k)\right\} \\
& +\frac{\xi}{96 \beta^{2}}\left\{k^{2} \mathrm{~T}_{1}^{\mu \nu, \alpha \beta}(u, k)-2 k^{2} \mathrm{~T}_{4}^{\mu \nu, \alpha \beta}(u, k)-\mathrm{T}_{8}^{\mu \nu, \alpha \beta}(u, k)+2 \mathrm{~T}_{13}^{\mu \nu, \alpha \beta}(u, k)\right\} \\
& +\pi \int \frac{d^{4} q}{(2 \pi)^{4}}\left\{\delta\left(q^{2}\right) n_{\beta}\left(\left|q^{o}\right|\right) \mathcal{P}\left[\frac{1}{(k+q)^{2}}\right]+\delta\left[(k+q)^{2}\right] n_{\beta}\left(\left|k^{o}+q^{o}\right|\right) \mathcal{P}\left[\frac{1}{q^{2}}\right]\right\} \mathrm{T}^{\mu \nu, \alpha \beta}(q, k),
\end{aligned}
$$

where $\mu$ is a simple redefinition of the renormalization parameter $\bar{\mu}$ given by $\mu \equiv \bar{\mu} \exp \left(\frac{23}{15}+\frac{1}{2} \ln 4 \pi-\frac{1}{2} \gamma\right)$, and the tensors $\mathrm{Q}^{\mu \nu, \alpha \beta}(k)$ and $\overline{\mathrm{Q}}^{\mu \nu, \alpha \beta}(k)$ are defined, respectively, by

$$
\begin{aligned}
\mathrm{Q}^{\mu \nu, \alpha \beta}(k)= & \frac{3}{2}\left\{\mathrm{~T}_{1}^{\mu \nu, \alpha \beta}(q, k)-\frac{1}{k^{2}} \mathrm{~T}_{8}^{\mu \nu, \alpha \beta}(q, k)+\frac{2}{k^{4}} \mathrm{~T}_{12}^{\mu \nu, \alpha \beta}(q, k)\right\} \\
& -\left[1-360\left(\xi-\frac{1}{6}\right)^{2}\right]\left\{\mathrm{T}_{4}^{\mu \nu, \alpha \beta}(q, k)+\frac{1}{k^{4}} \mathrm{~T}_{12}^{\mu \nu, \alpha \beta}(q, k)-\frac{1}{k^{2}} \mathrm{~T}_{13}^{\mu \nu, \alpha \beta}(q, k)\right\},
\end{aligned}
$$




$$
\overline{\mathrm{Q}}^{\mu \nu, \alpha \beta}(k)=\left[1+576\left(\xi-\frac{1}{6}\right)^{2}-60\left(\xi-\frac{1}{6}\right)\left(1-36 \xi^{\prime}\right)\right]\left\{\mathrm{T}_{4}^{\mu \nu, \alpha \beta}(q, k)+\frac{1}{k^{4}} \mathrm{~T}_{12}^{\mu \nu, \alpha \beta}(q, k)-\frac{1}{k^{2}} \mathrm{~T}_{13}^{\mu \nu, \alpha \beta}(q, k)\right\} .
$$

In the above and subsequent equations, we denote the coupling parameter in four dimensions $\xi(4)$ by $\xi$ and consequently $\xi^{\prime}$ means $d \xi(n) / d n$ evaluated at $n=4$. $\tilde{\mathrm{H}}^{\mu \nu, \alpha \beta}(k)$ is the complete contribution of a free massless quantum scalar field to the thermal graviton polarization tensor [3,4] and it is responsible for the instabilities found in flat spacetime at finite temperature [1,3, 1 . Eq. (17) reflects the fact that the kernel $\tilde{\mathrm{H}}^{\mu \nu, \alpha \beta}(k)$ has thermal as well as non-thermal contributions. Note that it reduces to the first term in the zero temperature limit $(\beta \rightarrow \infty)$

$$
\tilde{\mathrm{H}}^{\mu \nu, \alpha \beta}(k) \simeq-\frac{\alpha k^{4}}{4}\left\{\frac{1}{2} \ln \frac{\left|k^{2}\right|}{\mu^{2}} \mathrm{Q}^{\mu \nu, \alpha \beta}(k)+\frac{1}{3} \overline{\mathrm{Q}}^{\mu \nu, \alpha \beta}(k)\right\} .
$$

This asymptotic contribution to the CTP effective action includes both pure vacuum quantum fluctuations [28] and non-conformal fluctuations [30,31]. On the other hand, the leading term $\left(\beta^{-4}\right)$ at high temperature may be written as

$$
\tilde{\mathrm{H}}^{\mu \nu, \alpha \beta}(k) \simeq \frac{\pi^{2}}{30 \beta^{4}} \sum_{i=1}^{14} \mathrm{H}_{i}(r) \mathrm{T}_{i}^{\mu \nu, \alpha \beta}(u, K),
$$

where we have introduced the dimensionless external momentum $K^{\mu} \equiv k^{\mu} /|\vec{k}| \equiv(r, \hat{k})$. The $\mathrm{H}_{i}(r)$ coefficients were first given in [3] and generalized to the next-to-leading order $\left(\beta^{-2}\right)$ in $[4$. They have been reproduced with our sign conventions in Appendix $\mathrm{G}$ for completeness. It is important to note that the addition of the contribution of other kinds of matter fields, even graviton contributions, does not change the tensor structure of this leading contribution and only the overall factors are different [3]. However, this is not true for the subleading contributions as was shown in [4.

Finally, the noise kernel $\mathrm{N}^{\mu \nu, \alpha \beta}(x)$ and the dissipation kernel $\mathrm{D}^{\mu \nu, \alpha \beta}(x)$ are new and may be directly read off from (12) and (13), respectively. As expected the noise kernel appears in the imaginary part of the influence functional form of the CTP effective action and the dissipation kernel is the only antisymmetric kernel of the real part. Identification with the influence functional [15] provides a physical interpretation: $\mathrm{N}^{\mu \nu, \alpha \beta}(x)$ represents the random fluctuations of the plasma and $\mathrm{D}^{\mu \nu, \alpha \beta}(x)$ the dissipation of energy of the gravitational field.

\section{Einstein-Langevin equation}

In this section we show how a semiclassical Einstein-Langevin equation can be derived from the previous thermal CTP effective action. This equation depicts the stochastic evolution of the gravitational field under the influence of the fluctuations of the thermal plasma.

We first introduce the influence functional $\mathcal{F} \equiv \exp \left(i S_{I F}\right)$ which is defined from the influence action $S_{I F}$ of Feynman and Vernon 15. In fact, we can write this influence functional in terms of the the CTP effective action because in the semiclassical limit this effective action is completely equivalent to $S_{I F}$ [15, 36, 25,

$$
\mathcal{F}=\exp i\left(\operatorname{Re}\left\{\Gamma_{C T P}^{\beta}\left[h_{\mu \nu}^{ \pm}\right]\right\}+\frac{i}{2} \int d^{4} x d^{4} x^{\prime}\left[h_{\mu \nu}\right](x) \mathrm{N}^{\mu \nu, \alpha \beta}\left(x-x^{\prime}\right)\left[h_{\alpha \beta}\right]\left(x^{\prime}\right)\right),
$$

where $\operatorname{Re}\{\}$ denotes taking the real part. Following [15,25] 28], we can interpret the real part of the influence functional as the characteristic functional of a non-dynamical stochastic variable $j^{\mu \nu}(x)$,

$$
\Phi\left(\left[h_{\mu \nu}\right]\right)=\exp \left(-\frac{1}{2} \int d^{4} x d^{4} x^{\prime}\left[h_{\mu \nu}\right](x) \mathrm{N}^{\mu \nu, \alpha \beta}\left(x-x^{\prime}\right)\left[h_{\alpha \beta}\right]\left(x^{\prime}\right)\right) .
$$

This classical stochastic field represents probabilistically the quantum fluctuations of the matter field and is responsible for the dissipation of the gravitational field. By definition, the above characteristic functional is the functional Fourier transform of the probability distribution functional $\mathcal{P}\left[j^{\mu \nu}\right]$ with respect to $j^{\mu \nu}$, 


$$
\Phi\left(\left[h_{\mu \nu}\right]\right)=\int \mathcal{D} j^{\mu \nu} \mathcal{P}\left[j^{\mu \nu}\right] e^{i \int d^{4} x\left[h_{\mu \nu}\right](x) j^{\mu \nu}(x)} .
$$

Using (23) one can easily see that the probability distribution functional is related with the noise kernel by the formal expression,

$$
\mathcal{P}\left[j^{\mu \nu}\right]=\frac{\exp \left(-\frac{1}{2} \int d^{4} x d^{4} x^{\prime} j_{\mu \nu}(x)\left[\mathrm{N}^{\mu \nu, \alpha \beta}\left(x-x^{\prime}\right)\right]^{-1} j_{\alpha \beta}\left(x^{\prime}\right)\right)}{\int \mathcal{D} j^{\mu \nu} \exp \left(-\frac{1}{2} \int d^{4} x d^{4} x^{\prime} j_{\mu \nu}(x)\left[\mathrm{N}^{\mu \nu, \alpha \beta}\left(x-x^{\prime}\right)\right]^{-1} j_{\alpha \beta}\left(x^{\prime}\right)\right)} .
$$

For an arbitrary functional of the stochastic field $\mathcal{A}\left[j^{\mu \nu}\right]$, the average value with respect to the previous probability distribution functional is defined as the functional integral $\left\langle\mathcal{A}\left[j^{\mu \nu}\right]\right\rangle_{j} \equiv \int \mathcal{D}\left[j^{\mu \nu}\right] \mathcal{P}\left[j^{\mu \nu}\right] \mathcal{A}\left[j^{\mu \nu}\right]$. In terms of this stochastic average the influence functional can be written as $\mathcal{F}=\left\langle\exp \left(i \Gamma_{C T P}^{s t}\left[h_{\mu \nu}^{ \pm}\right]\right)\right\rangle_{j}$, where $\Gamma_{C T P}^{s t}\left[h_{\mu \nu}^{ \pm}\right]$is the modified effective action

$$
\Gamma_{C T P}^{s t}\left[h_{\mu \nu}^{ \pm}\right] \equiv \operatorname{Re}\left\{\Gamma_{C T P}^{\beta}\left[h_{\mu \nu}^{ \pm}\right]\right\}+\int d^{4} x\left[h_{\mu \nu}\right](x) j^{\mu \nu}(x)
$$

Clearly, because of the quadratic definition of the characteristic functional (23) and its relation with the probability distribution functional (24), the field $j^{\mu \nu}(x)$ is a zero mean Gaussian stochastic variable. This means that its two-point correlation function, which is given in terms of the noise kernel by

$$
\left\langle j^{\mu \nu}(x) j^{\alpha \beta}\left(x^{\prime}\right)\right\rangle_{j}=\mathrm{N}^{\mu \nu, \alpha \beta}\left(x-x^{\prime}\right),
$$

completely characterizes the stochastic process. The Einstein-Langevin equation follows from taking the functional derivative of the stochastic effective action (26) with respect to $\left[h_{\mu \nu}\right](x)$ and imposing $\left[h_{\mu \nu}\right](x)=0$ [34, 35, 25]. In our case, this leads to

$$
\frac{1}{\ell_{P}^{2}} \int d^{4} x^{\prime} \mathrm{L}_{(o)}^{\mu \nu, \alpha \beta}\left(x-x^{\prime}\right) h_{\alpha \beta}\left(x^{\prime}\right)+\frac{1}{2} T_{(\beta)}^{\mu \nu}+\int d^{4} x^{\prime}\left(\mathrm{H}^{\mu \nu, \alpha \beta}\left(x-x^{\prime}\right)-\mathrm{D}^{\mu \nu, \alpha \beta}\left(x-x^{\prime}\right)\right) h_{\alpha \beta}\left(x^{\prime}\right)+j^{\mu \nu}(x)=0 .
$$

To obtain a simpler and clearer expression we can rewrite this stochastic equation for the gravitational perturbation in the harmonic gauge $\bar{h}_{, \nu}^{\mu \nu}=0$,

$$
\square \bar{h}^{\mu \nu}(x)+\ell_{P}^{2}\left\{T_{(\beta)}^{\mu \nu}+2 P_{\rho \sigma, \alpha \beta} \int d^{4} x^{\prime}\left(\mathrm{H}^{\mu \nu, \alpha \beta}\left(x-x^{\prime}\right)-\mathrm{D}^{\mu \nu, \alpha \beta}\left(x-x^{\prime}\right)\right) \bar{h}^{\rho \sigma}\left(x^{\prime}\right)+2 j^{\mu \nu}(x)\right\}=0,
$$

where we have used the definition for $\bar{h}^{\mu \nu}(x)$ written in (5) and the tensor $P_{\rho \sigma, \alpha \beta}$ is given by

$$
P_{\rho \sigma, \alpha \beta}=\frac{1}{2}\left(\eta_{\rho \alpha} \eta_{\sigma \beta}+\eta_{\rho \beta} \eta_{\sigma \alpha}-\eta_{\rho \sigma} \eta_{\alpha \beta}\right)
$$

Note that this differential stochastic equation includes a non-local term responsible for the dissipation of the gravitational field and a noise source term which accounts for the fluctuations of the thermal plasma. They are connected by a FDR as described in the next section. Note also that this equation in combination with the correlation for the stochastic variable (27) determine the two-point correlation for the stochastic metric fluctuations $\left\langle\bar{h}_{\mu \nu}(x) \bar{h}_{\alpha \beta}\left(x^{\prime}\right)\right\rangle_{j}$ self-consistently.

\section{FLUCTUATION-DISSIPATION RELATION}

Now we want to see how the kernels found above to represent the noise and the dissipation of the system are functionally related. This FDR reflects the balance between the quantum fluctuations of the thermal plasma and the energy loss by the gravitational field. First, we explicitly see how this relation appears at zero temperature and compare with the results obtained in Refs. 28, 30. Secondly, we discuss in some detail the relation in the high temperature limit. Finally, using the properties of the thermal propagators, we formally show that the FDR is satisfied in general. 


\section{A. Fluctuation-dissipation relation at zero temperature}

Since the thermal functions $n_{\beta}\left(\left|q^{o}\right|\right)$ vanish at zero temperature, the complete zero temperature contribution to the noise and dissipation kernels can be directly read from (12) and (13)

$$
\begin{gathered}
\tilde{\mathrm{N}}_{(o)}^{\mu \nu, \alpha \beta}(k)=\pi^{2} \int \frac{d^{4} q}{(2 \pi)^{4}} \delta\left(q^{2}\right) \delta\left[(k+q)^{2}\right]\left\{\theta\left(k^{o}+q^{o}\right) \theta\left(-q^{o}\right)+\theta\left(-k^{o}-q^{o}\right) \theta\left(q^{o}\right)\right\} \mathrm{T}^{\mu \nu, \alpha \beta}(q, k), \\
\tilde{\mathrm{D}}_{(o)}^{\mu \nu, \alpha \beta}(k)=-i \pi^{2} \int \frac{d^{4} q}{(2 \pi)^{4}} \delta\left(q^{2}\right) \delta\left[(k+q)^{2}\right]\left\{\theta\left(k^{o}+q^{o}\right) \theta\left(-q^{o}\right)-\theta\left(-k^{o}-q^{o}\right) \theta\left(q^{o}\right)\right\} \mathrm{T}^{\mu \nu, \alpha \beta}(q, k) .
\end{gathered}
$$

The computation of these integrals is very simple because of the presence of the delta functions. Furthermore, since they are independent of the temperature the final tensor structure cannot depend on the four-velocity $u^{\mu}$ that defines the preferred co-moving coordinate system of the plasma. In fact, this simpler structure allow us to write all the integrals in terms of just only two integrals

$$
\int \frac{d^{4} q}{(2 \pi)^{4}} \delta\left(q^{2}\right) \delta\left[(k+q)^{2}\right]\left\{\theta\left(k^{o}+q^{o}\right) \theta\left(-q^{o}\right) \pm \theta\left(-k^{o}-q^{o}\right) \theta\left(q^{o}\right)\right\}=\frac{\theta\left(-k^{2}\right)\left[\theta\left(k^{o}\right) \pm \theta\left(-k^{o}\right)\right]}{4(2 \pi)^{3}},
$$

one corresponding to the noise kernel $(+\operatorname{sign})$ and the other to the dissipation kernel $(-\operatorname{sign})$. The final result for both kernels can be written in terms of the tensor $\mathrm{Q}^{\mu \nu, \alpha \beta}(k)$ defined in (18) as

$$
\begin{gathered}
\tilde{\mathrm{N}}_{(o)}^{\mu \nu, \alpha \beta}(k)=\left(\frac{\alpha \pi k^{4}}{4}\right) \theta\left(-k^{2}\right) \mathrm{Q}^{\mu \nu, \alpha \beta}(k), \\
\tilde{\mathrm{D}}_{(o)}^{\mu \nu, \alpha \beta}(k)=-i s g\left(k^{o}\right)\left(\frac{\alpha \pi k^{4}}{4}\right) \theta\left(-k^{2}\right) \mathrm{Q}^{\mu \nu, \alpha \beta}(k) .
\end{gathered}
$$

It is clear from these expressions, that the FDR satisfied by the noise and dissipation kernel at zero temperature is given by

$$
\tilde{\mathrm{N}}_{(o)}^{\mu \nu, \alpha \beta}(k)=-i s g\left(k^{o}\right) \tilde{\mathrm{D}}_{(o)}^{\mu \nu, \alpha \beta}(k) .
$$

This zero temperature result includes in one expression the FDR found in 28] for conformally coupled fields and that found in [30] for non-conformally coupled fields. We next discuss the pure thermal fluctuation contribution in the leading order at high temperature.

\section{B. Fluctuation-dissipation relation at high temperature}

Here, we describe in some detail the computation of the noise and dissipation kernels at high temperature in order to find the corresponding FDR. The computation of thermal integrals involving the thermal factors $n_{\beta}\left(\left|q^{o}\right|\right)$ is further complicated by the tensor structure of gravitation.

At high temperature one considers that the external momentum is much less than the temperature; that is $k^{o},|\vec{k}| \ll$ $\beta^{-1}$. Here we assume that the temperature is high enough so that this condition is satisfied, but remains well below the Planck temperature $\beta^{-1} \ll \beta_{P}^{-1} \sim \ell_{P}$ so that graviton loop corrections to the effective action may be neglected. On the other hand, the leading contribution can be obtained for internal momentum $|\vec{q}|$ of the order of the temperature $\beta^{-1}$ which means that the external momentum $|\vec{k}|$ is small compared with the internal momentum $|\vec{q}|$; i.e., $|\vec{k}| \ll|\vec{q}|$ (see, for example [49]). Let us start with the leading contribution $\left(\beta^{5}\right)$ for the noise kernel. The thermal contribution to the noise kernel $\tilde{\mathrm{N}}_{(\beta)}^{\mu \nu, \alpha \beta}(k)$ is given by the last three terms of Eq. (12) 


$$
\tilde{\mathrm{N}}_{(\beta)}^{\mu \nu, \alpha \beta}(k)=\pi^{2} \int \frac{d^{4} q}{(2 \pi)^{4}} \delta\left(q^{2}\right) \delta\left[(k+q)^{2}\right]\left\{n_{\beta}\left(\left|q^{o}\right|\right)+n_{\beta}\left(\left|k^{o}+q^{o}\right|\right)+2 n_{\beta}\left(\left|q^{o}\right|\right) n_{\beta}\left(\left|k^{o}+q^{o}\right|\right)\right\} \mathrm{T}^{\mu \nu, \alpha \beta}(q, k) .
$$

Integrating the $q^{o}$ variable, and taking into account the properties of the tensor $\mathrm{T}^{\mu \nu, \alpha \beta}(q, k)$ the above expression simplifies to

$$
\begin{aligned}
\tilde{\mathrm{N}}_{(\beta)}^{\mu \nu, \alpha \beta}(k)= & \frac{\pi}{2} \int \frac{d^{3} \vec{q}}{(2 \pi)^{3}} \frac{1}{2 q^{o}} \frac{1}{2 p^{o}}\left[n_{\beta}\left(q^{o}\right)+n_{\beta}\left(p^{o}\right)+2 n_{\beta}\left(q^{o}\right) n_{\beta}\left(p^{o}\right)\right] \\
& \times\left[\delta\left(p^{o}-q^{o}-k^{o}\right)+\delta\left(p^{o}+q^{o}+k^{o}\right)\right] \mathrm{T}^{\mu \nu, \alpha \beta}(q, k) \\
& +\{k \leftrightarrow-k\},
\end{aligned}
$$

where $q^{o}=|\vec{q}|$ and $p^{o}=|\vec{k}+\vec{q}|$. On dimensional grounds it is clear that the leading contribution at high temperature to the tensor $\mathrm{T}^{\mu \nu, \alpha \beta}(q, k)$ is given by its first term $\mathrm{T}_{3}^{\mu \nu, \alpha \beta}(q, k)$ only. We are also going to assume the following approximations:

$$
\begin{aligned}
p^{o} & \simeq q^{o}+\vec{k} \hat{q}, \\
p^{o}-q^{o}-k^{o} & \simeq-k^{o}+\vec{k} \hat{q}, \\
p^{o}+q^{o}+k^{o} & \simeq 2 q^{o} .
\end{aligned}
$$

Further simplifications arise because in this limit the thermal factor $n_{\beta}\left(p^{o}\right)$ may be approximated by

$$
n_{\beta}\left(p^{o}\right) \simeq n_{\beta}\left(q^{o}\right)+\vec{k} \hat{q} \frac{d n_{\beta}\left(q^{o}\right)}{d q^{o}} .
$$

For the particular combination of thermal functions in the noise kernel this yields

$$
n_{\beta}\left(q^{o}\right)+n_{\beta}\left(p^{o}\right)+2 n_{\beta}\left(q^{o}\right) n_{\beta}\left(p^{o}\right) \simeq 2 n_{\beta}\left(q^{o}\right)\left[1+n_{\beta}\left(q^{o}\right)\right] .
$$

As can be seen by a direct calculation the integrals with $\delta\left(p^{o}+q^{o}+k^{o}\right)$ do not contribute in this approximation. Then, the evaluation of the leading contribution at high temperature for the noise kernel (38) is reduced to calculate a solid angle integral and standard thermal integrals, separately,

$$
\tilde{\mathrm{N}}_{(\beta)}^{\mu \nu, \alpha \beta}(k)=\frac{\theta\left(k^{2}\right)}{16 \pi^{2}|\vec{k}|} \int d \Omega \delta(K Q) \mathrm{T}_{3}^{\mu \nu, \alpha \beta}(Q, K) \int_{0}^{\infty} d q^{o}\left(q^{o}\right)^{4} n_{\beta}\left(q^{o}\right)\left[1+n_{\beta}\left(q^{o}\right)\right] .
$$

In order to make the solid angle integral dimensionless we have used the external momentum $K$ defined previously and we have introduced a new lightlike vector $Q^{\mu} \equiv(1, \hat{q})$. Note that the theta function $\theta\left(k^{2}\right)$ appears as a condition imposed by the delta function in the solid angle integral. The explicit computation of these last two integrals in terms of the tensor basis $\mathrm{T}_{i}^{\mu \nu, \alpha \beta}(u, K)$ gives the final result to leading order in $\beta$

$$
\tilde{\mathrm{N}}_{(\beta)}^{\mu \nu, \alpha \beta}(k) \simeq \frac{\pi^{3}}{30 \beta^{5}} \frac{\theta\left(k^{2}\right)}{|\vec{k}|} \sum_{i=1}^{14} \mathrm{~N}_{i}(r) \mathrm{T}_{i}^{\mu \nu, \alpha \beta}(u, K),
$$

where the 14 coefficients $\mathrm{N}_{i}(r)$ are given by

$$
\begin{aligned}
\mathrm{N}_{i}(r)=\frac{1}{8}[ & K^{4}, 5 K^{6}, 35 K^{8}, K^{4}, 5 K^{6}, 5 K^{4} r, 35 K^{6} r,-\left(K^{2}-4 r^{2}\right) K^{2},-5\left(K^{2}-6 r^{2}\right) K^{4} \\
& \left.-5\left(K^{2}-6 r^{2}\right) K^{4},-5\left(3 K^{2}-4 r^{2}\right) K^{2} r, 3 K^{4}-24 K^{2} r^{2}+8 r^{4},-\left(K^{2}-4 r^{2}\right) K^{2}, 5 K^{4} r\right] .
\end{aligned}
$$

The determination of these coefficients involves the evaluation of the inverse of the cumbersome $14 \times 14$ matrix $M_{i j} \equiv \mathrm{T}_{i \mu \nu, \alpha \beta}(u, K) \mathrm{T}_{j}^{\mu \nu, \alpha \beta}(u, K)$ 3, 国. However, this can be easily performed with an algebraic computer program.

The thermal contribution to the dissipation kernel $\tilde{\mathrm{D}}_{(\beta)}^{\mu \nu, \alpha \beta}(k)$ can be obtained similarly. From the thermal terms of Eq. (13) we have 


$$
\tilde{\mathrm{D}}_{(\beta)}^{\mu \nu, \alpha \beta}(k)=-i \pi^{2} \int \frac{d^{4} q}{(2 \pi)^{4}} \delta\left(q^{2}\right) \delta\left[(k+q)^{2}\right]\left\{s g\left(k^{o}+q^{o}\right) n_{\beta}\left(\left|q^{o}\right|\right)-s g\left(q^{o}\right) n_{\beta}\left(\left|k^{o}+q^{o}\right|\right)\right\} \mathrm{T}^{\mu \nu, \alpha \beta}(q, k) .
$$

This integral can be simplified to

$$
\begin{aligned}
& \tilde{\mathrm{D}}_{(\beta)}^{\mu \nu, \alpha \beta}(k)=-\frac{i \pi}{2} \int \frac{d^{3} \vec{q}}{(2 \pi)^{3}} \frac{1}{2 q^{o}} \frac{1}{2 p^{o}}\left\{\delta\left(p^{o}-q^{o}-k^{o}\right)\left[n_{\beta}\left(q^{o}\right)-n_{\beta}\left(p^{o}\right)\right]\right. \\
& \left.-\delta\left(p^{o}+q^{o}+k^{o}\right)\left[n_{\beta}\left(q^{o}\right)+n_{\beta}\left(p^{o}\right)\right]\right\} \mathrm{T}^{\mu \nu, \alpha \beta}(q, k) \\
& -\{k \leftrightarrow-k\}
\end{aligned}
$$

Again the terms with $\delta\left(p^{o}+q^{o}+k^{o}\right)$ do not contribute to the final result. Using (40) to approximate the difference of thermal functions and following the above computation for the noise kernel, we can write the leading contribution in $\beta$ to the dissipation kernel as

$$
\tilde{\mathrm{D}}_{(\beta)}^{\mu \nu, \alpha \beta}(k) \simeq-i \frac{\pi^{3}}{60 \beta^{4}} \frac{\theta\left(k^{2}\right) k^{o}}{|\vec{k}|} \sum_{i=1}^{14} \mathrm{~N}_{i}(r) \mathrm{T}_{i}^{\mu \nu, \alpha \beta}(u, K),
$$

where $\mathrm{N}_{i}(r)$ are the coefficients given in (44). It is clear that these leading contributions for the noise and dissipation kernels satisfy a FDR at high temperature of the form

$$
\tilde{\mathrm{N}}_{(\beta)}^{\mu \nu, \alpha \beta}(k) \simeq i\left(\frac{2}{\beta k^{\circ}}\right) \tilde{\mathrm{D}}_{(\beta)}^{\mu \nu, \alpha \beta}(k) .
$$

It is important to note that the leading contribution for the noise kernel depends on $\beta^{-5}$ whereas that for the dissipation kernel depends on $\beta^{-4}$ and this is a direct consequence of the FDR. In contrast to the previous zero limit temperature FDR, this result may be interpreted to be a consequence of pure thermal fluctuations. We next show that formally there is a FDR valid to any order that includes both quantum and thermal fluctuations.

\section{Fluctuation-dissipation relation at finite temperature}

The existence of a FDR at finite temperature between the noise and dissipation kernels we have identified in the previous sections is very easy to prove under general conditions. We begin by showing that the FDR naively appears at the level of propagators as a direct consequence of the KMS relation 11] (see Appendix A). Then, using a generalization of this KMS relation, we see how the FDR is also satisfied by our noise and dissipation kernels.

To obtain the FDR at the level of propagators we need to introduce the Schwinger and the Hadamard propagators. These propagators are defined as the thermal average of the anticommutator $G\left(x-x^{\prime}\right) \equiv-i\left\langle\left[\phi(x), \phi\left(x^{\prime}\right)\right]\right\rangle_{\beta}$ and the commutator $G_{\beta}^{(1)}\left(x-x^{\prime}\right) \equiv-i\left\langle\left\{\phi(x), \phi\left(x^{\prime}\right)\right\}\right\rangle_{\beta}$, respectively. The first represents the linear response of a relativistic system to an external perturbation and the second the random fluctuations of the system itself [11, 12]. Since we can write the KMS condition satisfied by the propagators $G_{+-}^{\beta}$ and $G_{-+}^{\beta}$ in Fourier space as

$$
\tilde{G}_{-+}^{\beta}(k)=e^{\beta k^{o}} \tilde{G}_{+-}^{\beta}(k),
$$

the Fourier transform of both the Schwinger $\tilde{G}(k)$ and the Hadamard $\tilde{G}_{\beta}^{(1)}(k)$ propagators can be expressed, for example, in terms of $\tilde{G}_{+-}^{\beta}(k)$ alone

$$
\begin{aligned}
\tilde{G}(k) & \equiv \tilde{G}_{-+}^{\beta}(k)-\tilde{G}_{+-}^{\beta}(k)=\left(e^{\beta k^{o}}-1\right) \tilde{G}_{+-}^{\beta}(k), \\
\tilde{G}_{\beta}^{(1)}(k) & \equiv \tilde{G}_{-+}^{\beta}(k)+\tilde{G}_{+-}^{\beta}(k)=\left(e^{\beta k^{o}}+1\right) \tilde{G}_{+-}^{\beta}(k) .
\end{aligned}
$$

The FDR satisfied by these propagators follows inmediately from the above equalities 11. 12] 


$$
\tilde{G}_{\beta}^{(1)}(k)=\operatorname{coth}\left(\frac{\beta k^{o}}{2}\right) \tilde{G}(k) .
$$

Obviously, this relation can also be recovered if we write the explicit expressions for the Fourier transform of the propagators

$$
\begin{aligned}
\tilde{G}(k) & =-2 \pi i \operatorname{sg}\left(k^{o}\right) \delta\left(k^{2}\right), \\
\tilde{G}_{\beta}^{(1)}(k) & =-2 \pi i \operatorname{coth}\left(\frac{\beta\left|k^{o}\right|}{2}\right) \delta\left(k^{2}\right) .
\end{aligned}
$$

To use this last approach in our case could be a very difficult task because one needs to compute the integrals for the noise and dissipation kernels explicitly. On the other hand, if we follow the first technique we only need to generalize the KMS condition of Eq. (49) to product of two propagators. This generalization reads

$$
\tilde{G}_{-+}^{\beta}(k+q) \tilde{G}_{+-}^{\beta}(q)=e^{\beta k^{o}} \tilde{G}_{+-}^{\beta}(k+q) \tilde{G}_{-+}^{\beta}(q),
$$

and can be used to deduce the following formal identity

$$
\tilde{G}_{-+}^{\beta}(k+q) \tilde{G}_{+-}^{\beta}(q)+\tilde{G}_{+-}^{\beta}(k+q) \tilde{G}_{-+}^{\beta}(q)=\operatorname{coth}\left(\frac{\beta k^{o}}{2}\right)\left[\tilde{G}_{-+}^{\beta}(k+q) \tilde{G}_{+-}^{\beta}(q)-\tilde{G}_{+-}^{\beta}(k+q) \tilde{G}_{-+}^{\beta}(q)\right] .
$$

Finally, one only has to write, from the trace of Eq. (10) and the definitions $(12)$ and (13), the noise and dissipation kernels in terms of the propagators $\tilde{G}_{ \pm \mp}^{\beta}$, respectively, as

$$
\begin{aligned}
& \tilde{\mathrm{N}}^{\mu \nu, \alpha \beta}(k)=-\frac{1}{4} \int \frac{d^{4} q}{(2 \pi)^{4}}\left[\tilde{G}_{-+}^{\beta}(k+q) \tilde{G}_{+-}^{\beta}(q)+\tilde{G}_{+-}^{\beta}(k+q) \tilde{G}_{-+}^{\beta}(q)\right] \mathrm{T}^{\mu \nu, \alpha \beta}(q, k), \\
& \tilde{\mathrm{D}}^{\mu \nu, \alpha \beta}(k)=\frac{i}{4} \int \frac{d^{4} q}{(2 \pi)^{4}}\left[\tilde{G}_{-+}^{\beta}(k+q) \tilde{G}_{+-}^{\beta}(q)-\tilde{G}_{+-}^{\beta}(k+q) \tilde{G}_{-+}^{\beta}(q)\right] \mathrm{T}^{\mu \nu, \alpha \beta}(q, k),
\end{aligned}
$$

and use the formal equality (54) to prove that they are related by the thermal identity

$$
\tilde{\mathrm{N}}^{\mu \nu, \alpha \beta}(k)=i \operatorname{coth}\left(\frac{\beta k^{o}}{2}\right) \tilde{\mathrm{D}}^{\mu \nu, \alpha \beta}(k) .
$$

In coordinate space we have the analogous expression

$$
\mathrm{N}^{\mu \nu, \alpha \beta}(x)=\int d^{4} x^{\prime} \mathrm{K}_{F D}\left(x-x^{\prime}\right) \mathrm{D}^{\mu \nu, \alpha \beta}\left(x^{\prime}\right),
$$

where the fluctuation-dissipation kernel $\mathrm{K}_{F D}\left(x-x^{\prime}\right)$ is given by the integral

$$
\mathrm{K}_{F D}\left(x-x^{\prime}\right)=i \int \frac{d^{4} k}{(2 \pi)^{4}} e^{i k \cdot\left(x-x^{\prime}\right)} \operatorname{coth}\left(\frac{\beta k^{o}}{2}\right) .
$$

The proof of this FDR at finite temperature is in some sense formal because we have assumed along the argument that the integrals are always well defined. Nevertheless, the exact results obtained for the zero and high temperature limits indicate that the noise and dissipation kernels are well defined distributions [50]. The asymptotic analysis has also been useful to determine the physical origin of the fluctuations.

\section{LINEAR RESPONSE THEORY}

The purpose of this section is to understand the connection between the LRT [9 11, 51,52, and the functional methods we have used here. In the spirit of LRT the gravitational field is considered as a weak external source which imparts disturbances to the plasma whose response is studied to linear order. 
Let us first recall the main features of LRT. Consider a system described by the Hamiltonian operator $\hat{H}_{o}$ initially coupled to an external driving agent linearly, say $A_{\alpha}$. Since we are only interested in how the system responds to the external agent, and not the details of the agent, we will ignore the Hamiltonian for the external perturbation but write the complete operator Hamiltonian of the sytem as

$$
\hat{H}=\hat{H}_{o}+A_{\alpha} \hat{J}^{\alpha},
$$

where $J^{\alpha}$ is the current operator associated with the external agent. If the system is in thermal equilibrium before the external source is applied the first order response of the system to this external force is given by the thermal expectation value of the commutator of the current operator over its thermal average

$$
\left\langle\left[J^{\mu}(x)-\left\langle J^{\mu}(x)\right\rangle_{\beta}, J^{\nu}\left(x^{\prime}\right)-\left\langle J^{\nu}\left(x^{\prime}\right)\right\rangle_{\beta}\right]\right\rangle_{\beta} .
$$

In contrast, the intrinsic quantum fluctuations of the system are described by the thermal average of the anticommutator. In our case, the conserved current operator is given by the stress-energy tensor $T^{\mu \nu}(x)$ as derived from the classical action. The objective of this final section is to show that the response and fluctuation functions for the stress-energy tensor considered in the LRT are equivalent to our dissipation and noise kernels, respectively.

First, we write the classical action for the matter field to linear order in the gravitational perturbations,

$$
S_{m}\left[\phi, h_{\mu \nu}\right] \simeq \frac{1}{2} \int d^{4} x\left[\phi \square \phi+h_{\mu \nu} T^{\mu \nu}\right],
$$

with the stress-energy tensor given by

$$
T^{\mu \nu}=P^{\mu \nu, \alpha \beta} \partial_{\alpha} \phi \partial_{\beta} \phi+\xi\left(\eta^{\mu \nu} \square-\partial^{\mu} \partial^{\nu}\right) \phi^{2} .
$$

Note that $T^{\mu \nu}(x)$ is conserved if the classical unperturbed equation of motion for $\phi$ is satisfied and it reduces to the stress-energy tensor for a scalar field in flat spacetime if $\xi=0$. Alternatively, we can write the Hamiltonian formulation of our problem. If we introduce the conjugate momentum variable of the matter field to first order in the perturbation

$$
\Pi \equiv \frac{\partial \mathcal{L}}{\partial \dot{\phi}} \sim \dot{\phi}+\frac{1}{2} h_{\mu \nu} \frac{\partial T^{\mu \nu}}{\partial \dot{\phi}}
$$

the Hamiltonian can be written as

$$
H \simeq \frac{1}{2} \int d^{3} \vec{x}\left[\Pi^{2}+(\vec{\nabla} \phi)^{2}-h_{\mu \nu} T^{\mu \nu}\right] .
$$

Note that to first order $\dot{\phi}$ and $\Pi$ are interchangeable in the expression for the stress-energy tensor.

Using the thermal version of the Wick theorem [53,49], one can write, after some algebra, the equilibrium thermal average of the two-point function for the stress-energy tensor operator at different spacetime points in terms of products of thermal propagators,

$$
\left\langle T^{\mu \nu}(x) T^{\alpha \beta}\left(x^{\prime}\right)\right\rangle_{\beta}-\left\langle T^{\mu \nu}(x)\right\rangle_{\beta}\left\langle T^{\alpha \beta}\left(x^{\prime}\right)\right\rangle_{\beta}=-2 \int \frac{d^{4} k}{(2 \pi)^{4}} e^{i k \cdot\left(x-x^{\prime}\right)} \int \frac{d^{4} q}{(2 \pi)^{4}} \tilde{G}_{-+}^{\beta}(k+q) \tilde{G}_{+-}^{\beta}(q) \mathrm{T}^{\mu \nu, \alpha \beta}(q, k) .
$$

Finally, defining $\Delta_{\beta} T^{\mu \nu}(x) \equiv T^{\mu \nu}(x)-\left\langle T^{\mu \nu}(x)\right\rangle_{\beta}$ and using the expressions for the the noise and dissipation kernels given in (55) and (56) respectively, we obtain

$$
\begin{aligned}
& \left\langle\left\{\Delta_{\beta} T^{\mu \nu}(x), \Delta_{\beta} T^{\alpha \beta}\left(x^{\prime}\right)\right\}\right\rangle_{\beta}=8 \mathrm{~N}^{\mu \nu, \alpha \beta}\left(x-x^{\prime}\right), \\
& \left\langle\left[\Delta_{\beta} T^{\mu \nu}(x), \Delta_{\beta} T^{\alpha \beta}\left(x^{\prime}\right)\right]\right\rangle_{\beta}=8 i \mathrm{D}^{\mu \nu, \alpha \beta}\left(x-x^{\prime}\right) .
\end{aligned}
$$

From these formal identities we conclude that the functional method gives a description of the lowest order dynamics of a near-equilibrium system equivalent to that given traditionally by the LRT. 


\section{CONCLUSIONS}

In this paper we show how the functional methods can be used effectively to study the non-equilibrium dynamics of a thermal quantum field in an external gravitational field. The close time path (CTP) effective action and the influcence functional were used to derive the noise and dissipation kernels of the open system. We show the formal equivalence of this method with the traditional linear response theory (LRT) for lowest order perturbance of a near-equilibrium system, and how the response functions such as the contribution of the quantum scalar field to the thermal graviton polarization tensor can be derived. An important quantity not usually obtained in LRT approaches but of equal manifest importance in the CTP approach is the noise term arising from the quantum and thermal fluctuations in the plasma field. With this we derived a Langevin-type equation for the non-equilibrium dynamics of the gravitational field under the influence of the plasma. The back reaction of the plasma on the gravitational field is embodied in a fluctuation-dissipation relation (FDR), which connects the quantum fluctuations of the thermal plasma and the energy dissipated by the external gravitational field. We formally prove the existence of such a relation for thermal plasmas at all temperatures.

This powerful method perfected in recent years has been successfully applied to semiclassical gravity and cosmological problems by a number of authors. It should also prove useful for thermal field theory problems in QED and QCD, such as electroweak phase transition, quark-gluon plasma and disoriented chiral condensates in heavy-ion collision processes.

\section{ACKNOWLEDGMENTS}

The work of A.C. was supported in part by the CICYT research Project No. AEN95-0882, the Comissionat per a Universitats i Recerca under a cooperative agreement between MIT and Generalitat de Catalunya and funds provided by the U.S. Department of Energy (D.O.E.) under cooperative research agreement DE-FC02-94ER40818. BLH was supported in part by NSF grant PHY94-21849.

\section{APPENDIX A: THERMAL FIELD THEORY}

In this Appendix, we describe the CTP formalism in flat spacetime in order to compute the effective action for a scalar field in thermal equilibrium [35,53 55]. Using the CTP formalism one can deal with non-equilibrium situations because we do not have to assume anything about the final state of the system in contrast to other formalisms of quantum field theory at finite temperature. [56,57,49]. We begin with a brief general description of the CTP formalism

for an arbitrary initial state. Then, we explain with more detail the particular features of the formalism for an initial state in thermal equilibrium.

\section{Arbitrary initial state}

The CTP formalism is a functional technique to obtain expectation values of products of quantum field operators [13] and dynamical equations for the mean field which are real and causal 47, 48]. For simplicity, consider a scalar field $\Phi(x)$ described initially by the time independent Hamiltonian $H[\Phi(x)]$. Suppose that an external classical source $J(x)$ is turned on and the perturbed Hamiltonian assumes the form $H+J(x) \Phi(x)$. If the initial quantum state of the 
field is defined by the density matrix operator $\rho$, we can define the CTP generating functional, $Z_{\rho}[J]$, as the following statistical average over the initial quantum state [14, 35, 38, 44,

$$
Z_{\rho}[J] \equiv \operatorname{Tr}\left\{\rho T_{C} e^{i \int_{C} d^{n} x J(x) \phi(x)}\right\} .
$$

In the above definition $\phi(x)$ is the scalar field operator which evolves in time under the unperturbed Hamiltonian $H$, $n$ is the number of spacetime dimensions, $T_{C}$ is the time ordering operator defined over a contour $C$ in complex time which goes forward in time and then back to the initial point (see Fig. 1) and the integral symbol $\int_{C}$ means complex integration along this path. Note that in this picture the time evolution of the initial state is determined by the external source $J(x)$ alone. Our main concern is to apply this formalism to a system initially in thermal equilibrium.

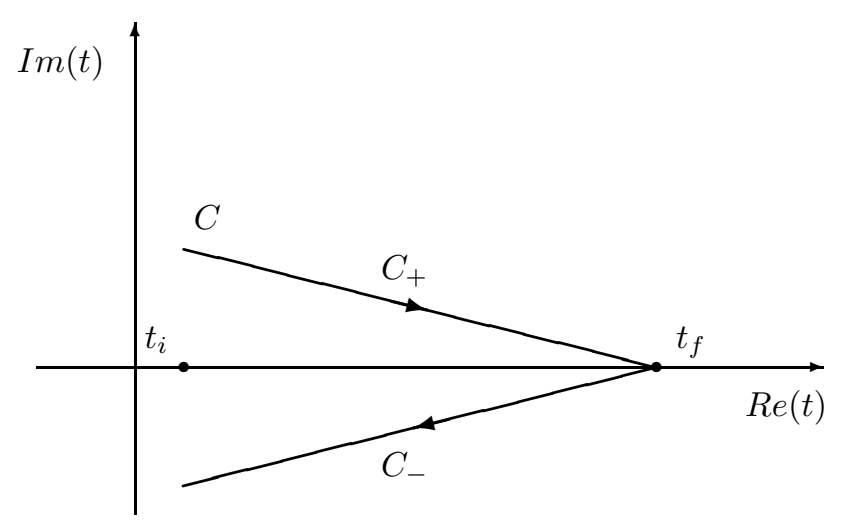

FIG. 1. Integration contour $C$ in the complex time plane for the CTP formalism of non-equilibrium quantum fields. This path can be splitted in two separated segments $C_{+} \cup C_{-}$, the first going forward in time and the second backward. The small deviation from the real axis is needed to ensure a well defined path integral representation of the generating functional.

By differentiating with respect to the external source and then taking $J=0$ this functional generates the expectation value over the initial quantum state specified by the density matrix $\rho$ of time ordered products of the field operator along the contour $C$. In particular, the two-point function is given by,

$$
\left.\frac{1}{Z_{\rho}[0]} \frac{\delta^{2} Z_{\rho}[J]}{i \delta J(x) i \delta J\left(x^{\prime}\right)}\right|_{J=0}=\frac{\operatorname{Tr}\left\{\rho T_{C} \phi(x) \phi\left(x^{\prime}\right)\right\}}{\operatorname{Tr}\{\rho\}} .
$$

To write an integral representation of the generating functional we have to introduce a complete basis of eigenstates $\left\{\left|\varphi, t_{i}\right\rangle\right\}$ of the field operator $\phi(x)$ at the initial time $t_{i}$, i.e. $\phi\left(t_{i}, \vec{x}\right)\left|\varphi, t_{i}\right\rangle=\varphi(\vec{x})\left|\varphi, t_{i}\right\rangle$ where $\varphi(\vec{x})$ is the eigenvalue. This representation for $Z_{\rho}[J]$ is

$$
Z_{\rho}[J]=\int d[\varphi] d\left[\varphi^{\prime}\right]\left\langle\varphi, t_{i}|\rho| \varphi^{\prime}, t_{i}\right\rangle\left\langle\varphi^{\prime}, t_{i}\left|T_{C} e^{i \int_{C} d^{n} x J(x) \phi(x)}\right| \varphi, t_{i}\right\rangle .
$$

The last term in the integral may be interpreted as a transition amplitude from the initial state $\left|\varphi, t_{i}\right\rangle$ to another initial state $\left|\varphi^{\prime}, t_{i}\right\rangle$ following the close time path $C$, and then, it can be expressed as a path integral with the appropriate boundary conditions,

$$
Z_{\rho}[J]=\int d[\varphi] d\left[\varphi^{\prime}\right]\left\langle\varphi, t_{i}|\rho| \varphi^{\prime}, t_{i}\right\rangle \int_{\phi\left(t_{i}\right)=\varphi}^{\phi\left(t_{i}\right)=\varphi^{\prime}} \mathcal{D}[\phi] e^{i \int_{C} d^{n} x(\mathcal{L}[\phi(x)]+J(x) \phi(x))} .
$$

In the above equation, $\mathcal{L}[\phi(x)]$ is the Lagrangian density corresponding to the unperturbed Hamiltonian of the scalar field theory. Now, as usual, we introduce the generating functional $W_{\rho}[J] \equiv-i \ln Z_{\rho}[J]$ that generates the connected part of the $n$-point functions and define the CTP effective action as its Legendre transform, 


$$
\Gamma_{\rho}^{C T P}[\bar{\phi}] \equiv W_{\rho}[J]-\int_{C} d^{n} x J(x) \bar{\phi}(x),
$$

where $\bar{\phi}(x)$ is the expectation value of the field $\phi(x)$ over the initial state in the presence of the external source $J(x)$,

$$
\bar{\phi}(x) \equiv \frac{\delta W_{\rho}[J]}{\delta J(x)} \equiv \frac{\operatorname{Tr}\left\{\rho T_{C} \phi(x) e^{i \int_{C} d^{n} x^{\prime} J\left(x^{\prime}\right) \phi\left(x^{\prime}\right)}\right\}}{\operatorname{Tr}\left\{\rho T_{C} e^{i \int_{C} d^{n} x^{\prime} J\left(x^{\prime}\right) \phi\left(x^{\prime}\right)}\right\}} .
$$

Note that $\bar{\phi}$ becomes the mean value of the field if we substitute $J=0$. The CTP effective action is the generating functional of one-particle irreducible graphs and contains all the quantum corrections to the classical action. Since we have only needed information about the initial state of the system a real dynamical equation with causal boundary conditions can be derived from this effective action .

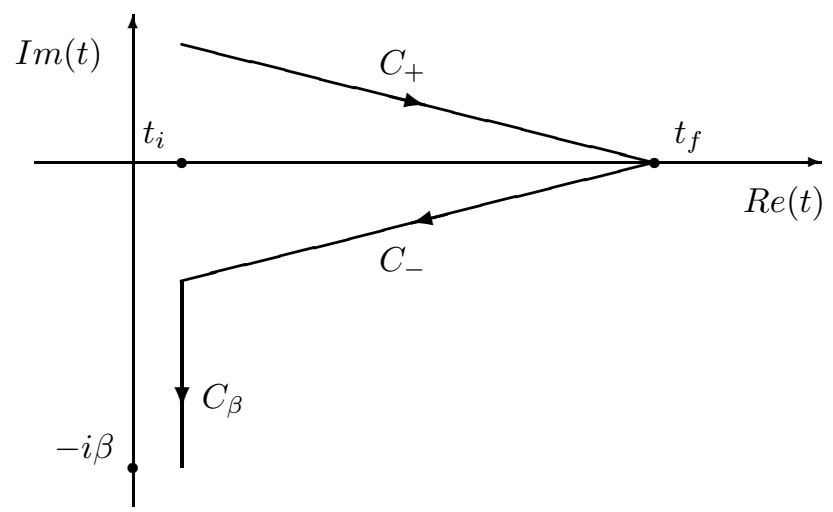

FIG. 2. Integration contour in the complex time plane for the CTP path integral useful for a system with an initial state in thermal equilibrium.

\section{Initial state in thermal equilibrium}

For practical purposes, we are going to describe in some detail how to obtain the Green functions and the effective action for a quantum system with an initial state in thermal equilibrium at temperature $\beta^{-1}$ [54, 38, 55]. Consider the Lagrangian density for a free massive scalar field

$$
\mathcal{L}[\phi(x)]=-\frac{1}{2}\left(\partial_{\mu} \phi \partial^{\mu} \phi+m^{2} \phi^{2}\right) .
$$

The initial thermal state of this system is described by a normalized density matrix of the form $\rho_{\beta}=e^{-\beta H} / \operatorname{Tr}\left\{e^{-\beta H}\right\}$, where $H$ is the Hamiltonian operator corresponding to (A7) at some initial time $t_{i}$; i.e., the unperturbed Hamiltonian of the system before the external source $J(x)$ is connected. In this case, the path integral representation of the generating functional given in Eq. (A4) can be written as

$$
Z_{\beta}[J]=\frac{1}{\operatorname{Tr}\left\{e^{-\beta H}\right\}} \int d[\varphi] d\left[\varphi^{\prime}\right]\left\langle\varphi, t_{i}-i \beta \mid \varphi^{\prime}, t_{i}\right\rangle \int_{\phi\left(t_{i}\right)=\varphi}^{\phi\left(t_{i}\right)=\varphi^{\prime}} \mathcal{D}[\phi] e^{i \int_{C} d^{n} x(\mathcal{L}[\phi(x)]+J(x) \phi(x))} .
$$

In deriving this expression, we have used the fact that the density matrix operator $\rho_{\beta}$ can be seen as a time translation operator in the complex plane because the unperturbed Hamiltonian is the generator of time evolution for the eigenstates of the field operator $\phi(x)$. In other words, since the field transforms as $\phi(t, \vec{x})=e^{i t H} \phi(0, \vec{x}) e^{-i t H}$ and the eigenstates evolve in time simply as $|\varphi, t\rangle=e^{i t H}|\varphi, 0\rangle$, the density matrix generates a translation in complex time $t \rightarrow t-i \beta$. The above representation for $Z_{\beta}[J]$ may be written in a more compact form as 


$$
Z_{\beta}[J]=\mathcal{N} \int \mathcal{D}[\phi] e^{i \int_{C} d^{n} x(\mathcal{L}[\phi(x)]+J(x) \phi(x))} .
$$

The path integral is defined over all possible field configurations along the time path $C$ with initial boundary condition $\phi\left(t_{i}\right)=\phi\left(t_{i}-i \beta\right)$ and the normalization term $\mathcal{N}$ includes all the factors which are independent of the external source $J(x)$. We would be also able to write the above compact expression for the path integral along the time path in the complex plane of Fig. 2. But, the contribution to the path integral along the segment $C_{\beta}$ has no dynamical consequences in our case because the external source is zero along this vertical segment. In the usual path integral approach to thermal field theory [56,57, 49] (see also [54]), the external source is also non zero along $C_{\beta}$ by definition in contrast with our approach $38,43,55$. However, it can be justified that both are consistent asymtotically; that is, when $t_{i} \rightarrow-\infty$ and $t_{f} \rightarrow \infty$. We are going to assume this condition from now on.

To perform the path integral of the generating functional it is convenient to make the change of field variables $\phi(x) \rightarrow \phi(x)-\int_{C} d^{n} x^{\prime} G_{\beta}^{C}\left(x-x^{\prime}\right) J\left(x^{\prime}\right)$, where the propagator $G_{\beta}^{C}\left(x-x^{\prime}\right)$ satisfies the differential equation,

$$
\left(\square_{C}-m^{2}\right) G_{\beta}^{C}\left(x-x^{\prime}\right)=\delta_{C}\left(x-x^{\prime}\right) .
$$

The subscript $C$ in the d'Alambertian $\square_{C}$ and the delta function $\delta_{C}$ means that these objects are defined along the complex path $C$. The subscript $\beta$ in $G_{\beta}^{C}$ is written to remind that this propagator has to be constructed with the appropriate thermal boundary conditions. If we redefine the normalization factor $\mathcal{N}$ by $Z_{\beta}[0]$ in order to include a new path integral independent of $J(x)$, the change of variable yields

$$
Z_{\beta}[J]=Z_{\beta}[0] e^{-\frac{i}{2} \int_{C} d^{n} x d^{n} x^{\prime} J(x) G_{\beta}^{C}\left(x-x^{\prime}\right) J\left(x^{\prime}\right)} .
$$

It is clear from the thermal version of (A1) and the above expression for the generating functional that the propagator $G_{\beta}^{C}\left(x-x^{\prime}\right)$ can be interpreted as the thermal average of two time-ordered operators along $C$

$$
G_{\beta}^{C}\left(x-x^{\prime}\right)=-i\left\langle T_{C} \phi(x) \phi\left(x^{\prime}\right)\right\rangle_{\beta} .
$$

Above we have introduced the shorthand notation $\langle A\rangle_{\beta} \equiv \operatorname{Tr}\left\{\rho_{\beta} A\right\}$ for the trace of a general operator $A$ with respect to the thermal density operator. It does not take more additional work at this point to show that the Wick theorem also applies at finite temperature [53,49]. Let us find an explicit expression for the thermal propagator. Using the step function $\theta_{C}$, defined also along the contour $C$, one can decompose the propagator as

$$
G_{\beta}^{C}\left(\tau-\tau^{\prime} ; \vec{x}-\vec{x}^{\prime}\right)=\theta_{C}\left(\tau-\tau^{\prime}\right) G_{\beta}^{+}\left(\tau-\tau^{\prime} ; \vec{x}-\vec{x}^{\prime}\right)+\theta_{C}\left(\tau^{\prime}-\tau\right) G_{\beta}^{-}\left(\tau-\tau^{\prime} ; \vec{x}-\vec{x}^{\prime}\right),
$$

where we have denoted the time variable along the path $C$ by $\tau$ and defined the propagators $G_{\beta}^{+}\left(\tau-\tau^{\prime} ; \vec{x}-\vec{x}^{\prime}\right)=$ $G_{\beta}^{-}\left(\tau^{\prime}-\tau ; \vec{x}^{\prime}-\vec{x}\right) \equiv-i\left\langle\phi(\tau ; \vec{x}) \phi\left(\tau^{\prime} ; \vec{x}^{\prime}\right)\right\rangle_{\beta}$. The trace nature of the generating functional and the Hamiltonian time evolution of the field operator $\phi(x)$ impose some relations on certain propagators. For example, they lead to equalities like $G_{\beta}^{-}(\tau ; \vec{x})=G_{\beta}^{+}(\tau-i \beta ; \vec{x})$ which are generally known as Kubo-Martin-Schwinger (KMS) relations 10,11. In fact, these conditions are a direct consequences of thermal equilibrium and are necessary to preserve the periodic boundary condition in the change of field variable previously performed in order to simplify the path integral representation of the generating functional [58,59]. The solution of (A10), compatible with the KMS relation, may be expressed in terms of the thermal function $n_{\beta}\left(p^{o}\right)=\left(e^{\beta p^{o}}-1\right)^{-1}$ as

$$
G_{\beta}^{C}\left(\tau-\tau^{\prime} ; \vec{x}-\vec{x}^{\prime}\right)=\int \frac{d^{n} p}{(2 \pi)^{n}} e^{i p \cdot\left(x-x^{\prime}\right)}\left[\theta_{C}\left(\tau-\tau^{\prime}\right)+n_{\beta}\left(p^{o}\right)\right](-2 \pi i) s g\left(p^{o}\right) \delta\left(p^{2}+m^{2}\right) .
$$

Now, for simplicity in actual computations, it is convenient to introduce a matrix notation for the thermal propagator $G_{\beta}^{C}$ which takes into account the segment of the path, $C_{+}$or $C_{-}$, where the time variable is choosen to lie. We have four different possibilities: 
- if $\tau, \tau^{\prime} \in C_{+}$or $\tau, \tau^{\prime} \in C_{-}$we may write, respectively,

$$
G_{ \pm \pm}^{\beta}\left(x-x^{\prime}\right)=\int \frac{d^{n} p}{(2 \pi)^{n}} e^{i p \cdot\left(x-x^{\prime}\right)}\left[\frac{\mp 1}{p^{2}+m^{2} \mp i \epsilon}-2 \pi i n_{\beta}\left(\left|p^{o}\right|\right) \delta\left(p^{2}+m^{2}\right)\right]
$$

- if $\tau \in C_{+}$and $\tau^{\prime} \in C_{-}$, or vice versa, then respectively,

$$
G_{ \pm \mp}^{\beta}\left(x-x^{\prime}\right)=\int \frac{d^{n} p}{(2 \pi)^{n}} e^{i p \cdot\left(x-x^{\prime}\right)}(-2 \pi i)\left[\theta\left(\mp p^{o}\right)+n_{\beta}\left(\left|p^{o}\right|\right)\right] \delta\left(p^{2}+m^{2}\right) .
$$

Since the time variable in the above equations takes values on the real axis alone, it is very easy to see that all these propagator components may be expressed as thermal averages

$$
\begin{aligned}
& G_{++}^{\beta}\left(x-x^{\prime}\right)=-i\left\langle T \phi(x) \phi\left(x^{\prime}\right)\right\rangle_{\beta} \\
& G_{--}^{\beta}\left(x-x^{\prime}\right)=-i\left\langle\bar{T} \phi(x) \phi\left(x^{\prime}\right)\right\rangle_{\beta} \\
& G_{-+}^{\beta}\left(x-x^{\prime}\right)=-i\left\langle\phi(x) \phi\left(x^{\prime}\right)\right\rangle_{\beta}=G_{+-}^{\beta}\left(x^{\prime}-x\right),
\end{aligned}
$$

where $T$ and $\bar{T}$ are the usual time and anti-time ordering operators, respectively. If we define in the same way the external sources $J_{ \pm}(t, \vec{x}) \equiv J(\tau, \vec{x})$ for $\tau \in C_{ \pm}$, we can write the generating functional in matrix form

$$
Z_{\beta}\left[J_{a}\right]=Z_{\beta}[0] e^{-\frac{i}{2} \int d^{n} x d^{n} x^{\prime} J_{a}^{T}(x) G_{a b}^{\beta}\left(x-x^{\prime}\right) J_{b}\left(x^{\prime}\right)} .
$$

In the exponents, the time integrals range from $-\infty$ to $+\infty$, the subindixes $a, b$ take values,+- and the transposed source vector is defined by $J_{a}^{T}(x) \equiv\left(J_{+}(x),-J_{-}(x)\right)$. The minus sign in the second component of the source vector is a relic to the fact that originally the integral for the time variable along the segment $C_{-}$was from $+\infty$ to $-\infty$. If we were interested to specify initial vacuum boundary conditions $\left(\rho_{o} \equiv \mid 0\right.$, in $\rangle\langle 0$, in $\left.|\right)$ instead of thermal equilibrium conditions we could still use A18 substituting the thermal propagator $G_{a b}^{\beta}$ by the vacuum propagator $G_{a b}^{o} \equiv \lim _{\beta \rightarrow \infty} G_{a b}^{\beta}$ (see, for example $37,42,55$ ).

Finally, we are interested to obtain the CTP effective action at finite temperature in terms of the propagator. Following [42], where the derivation of the CTP effective action in the case of an initial pure vacuum state was explained in some detail, but taking into account the particular boundary conditions for the thermal case, we arrive to the expression (see also 55,43 )

$$
\Gamma_{C T P}^{\beta}\left[\bar{\phi}_{a}\right]=S\left[\bar{\phi}_{a}\right]-\frac{i}{2} \operatorname{Tr}\left\{\ln G_{a b}^{\beta}\right\}
$$

where $S\left[\bar{\phi}_{a}\right]$ is the classical action for the scalar field evaluated at the expectation value of $\phi_{a}(x)$ in the presence of the external source $J_{a}(x)$.

\section{CTP effective action for two fields in the semiclassical approximation}

We now derive the CTP effective action at finite temperature for two fields in the semiclassical approximation in order to generalize Eq. (A19) and make it of direct applicability to our problem. For simplicity, consider two different scalar fields described by the general Lagrangian density

$$
\mathcal{L}[\phi(x)]+\mathcal{L}[\psi(x), \phi(x)]
$$

where the second term is assumed to be quadratic in the field $\psi(x)$. In our case, the first part, $\mathcal{L}[\phi(x)]$, will represent the Lagrangian density of the gravitational field, and the second part, the Lagrangian density of a thermal scalar field 
coupled to gravity. Since, we are only interested in the dynamics of the gravitational field we perturb the system by an external source coupled only to $\phi(x)$. The CTP generating functional for this system will be

$$
e^{i W_{\beta}\left[J_{ \pm}\right]} \equiv \int \mathcal{D}[\phi] \mathcal{D}[\psi] e^{i \int_{C} d^{n} x(\mathcal{L}[\phi(x)]+\mathcal{L}[\psi(x), \phi(x)]+J(x) \phi(x))},
$$

where $C$ is the time integration path of Fig. 1 and the field $\psi(x)$ has the appropriate thermal boundary conditions. If one of the fields is assumed to evolve classically, in our case $\phi(x)$, the above CTP generating functional may be approximated by

$$
e^{i W_{\beta}\left[J_{ \pm}\right]} \simeq e^{i \int_{C} d^{n} x(\mathcal{L}[\bar{\phi}(x)]+J(x) \bar{\phi}(x))} \int \mathcal{D}[\psi] e^{i \int_{C} d^{n} x \mathcal{L}[\psi(x), \bar{\phi}(x)]},
$$

where $\bar{\phi}(x)$ is the classical field written in terms of $J(x)$. The remaining path integral is in fact a functional of $\bar{\phi}(x)$ alone and can be identified with the influence functional of Feynman and Vernon [15]. Since $\mathcal{L}[\psi(x), \bar{\phi}(x)]$ has been assumed to be quadratic in $\psi(x)$, the path integral is Gaussian and may be performed exactly. In matrix notation we have

$$
\exp \left(-\frac{i}{2} \operatorname{Tr}\left\{\ln \bar{G}_{a b}^{\beta}\left[\bar{\phi}_{ \pm}\right]\right\}\right)
$$

where $\bar{G}_{a b}^{\beta}\left[\bar{\phi}_{ \pm}\right]$is the corresponding matrix propagator with thermal boundary conditions of the Lagrangian density $\mathcal{L}[\psi(x), \bar{\phi}(x)]$. When this result is substituted in the CTP generating functional and the Legendre transform with respect $J(x)$ is done, we can finally write the thermal CTP effective action as

$$
\Gamma_{C T P}^{\beta}\left[\bar{\phi}_{ \pm}\right]=S\left[\bar{\phi}_{+}\right]-S\left[\bar{\phi}_{-}\right]-\frac{i}{2} \operatorname{Tr}\left\{\ln \bar{G}_{a b}^{\beta}\left[\bar{\phi}_{ \pm}\right]\right\} .
$$

This generalized expression for the CTP effective action justifies the use of Eq. (7).

\section{APPENDIX B: TENSOR BASIS}

The complicated tensor structure of the thermal CTP effective action may be simplified if the following tensor basis is used

$$
\begin{aligned}
\mathrm{T}_{1}^{\mu \nu, \alpha \beta}(X, Y)= & \eta^{\alpha \nu} \eta^{\beta \mu}+\eta^{\alpha \mu} \eta^{\beta \nu} \\
\mathrm{T}_{2}^{\mu \nu, \alpha \beta}(X, Y)= & X^{\mu} X^{\beta} \eta^{\alpha \nu}+X^{\mu} X^{\alpha} \eta^{\beta \nu}+X^{\nu} X^{\beta} \eta^{\alpha \mu}+X^{\nu} X^{\alpha} \eta^{\beta \mu} \\
\mathrm{T}_{3}^{\mu \nu, \alpha \beta}(X, Y)= & X^{\alpha} X^{\beta} X^{\mu} X^{\nu} \\
\mathrm{T}_{4}^{\mu \nu, \alpha \beta}(X, Y)= & \eta^{\mu \nu} \eta^{\alpha \beta} \\
\mathrm{T}_{5}^{\mu \nu, \alpha \beta}(X, Y)= & X^{\mu} X^{\nu} \eta^{\alpha \beta}+X^{\alpha} X^{\beta} \eta^{\mu \nu} \\
\mathrm{T}_{6}^{\mu \nu, \alpha \beta}(X, Y)= & X^{\beta}\left(Y^{\nu} \eta^{\alpha \mu}+Y^{\mu} \eta^{\alpha \nu}\right)+X^{\alpha}\left(Y^{\nu} \eta^{\beta \mu}+Y^{\mu} \eta^{\beta \nu}\right) \\
& +Y^{\beta}\left(X^{\nu} \eta^{\alpha \mu}+X^{\mu} \eta^{\alpha \nu}\right)+Y^{\alpha}\left(X^{\nu} \eta^{\beta \mu}+X^{\mu} \eta^{\beta \nu}\right) \\
\mathrm{T}_{7}^{\mu \nu, \alpha \beta}(X, Y)= & Y^{\nu} X^{\alpha} X^{\beta} X^{\mu}+Y^{\mu} X^{\alpha} X^{\beta} X^{\nu}+Y^{\beta} X^{\alpha} X^{\mu} X^{\nu}+Y^{\alpha} X^{\beta} X^{\mu} X^{\nu} \\
\mathrm{T}_{8}^{\mu \nu, \alpha \beta}(X, Y)= & Y^{\beta} Y^{\nu} \eta^{\alpha \mu}+Y^{\beta} Y^{\mu} \eta^{\alpha \nu}+Y^{\alpha} Y^{\nu} \eta^{\beta \mu}+Y^{\alpha} Y^{\mu} \eta^{\beta \nu} \\
\mathrm{T}_{9}^{\mu \nu, \alpha \beta}(X, Y)= & Y^{\mu} Y^{\nu} X^{\alpha} X^{\beta}+Y^{\alpha} Y^{\beta} X^{\mu} X^{\nu} \\
\mathrm{T}_{10}^{\mu \nu, \alpha \beta}(X, Y)= & \left(Y^{\beta} X^{\alpha}+Y^{\alpha} X^{\beta}\right)\left(Y^{\nu} X^{\mu}+Y^{\mu} X^{\nu}\right) \\
\mathrm{T}_{11}^{\mu \nu, \alpha \beta}(X, Y)= & Y^{\beta} Y^{\mu} Y^{\nu} X^{\alpha}+Y^{\alpha} Y^{\mu} Y^{\nu} X^{\beta}+Y^{\alpha} Y^{\beta} Y^{\nu} X^{\mu}+Y^{\alpha} Y^{\beta} Y^{\mu} X^{\nu} \\
\mathrm{T}_{12}^{\mu \nu, \alpha \beta}(X, Y)= & Y^{\alpha} Y^{\beta} Y^{\mu} Y^{\nu} \\
\mathrm{T}_{13}^{\mu \nu, \alpha \beta}(X, Y)= & Y^{\mu} Y^{\nu} \eta^{\alpha \beta}+Y^{\alpha} Y^{\beta} \eta^{\mu \nu} \\
\mathrm{T}_{14}^{\mu \nu, \alpha \beta}(X, Y)= & \left(Y^{\nu} X^{\mu}+Y^{\mu} X^{\nu}\right) \eta^{\alpha \beta}+\left(Y^{\beta} X^{\alpha}+Y^{\alpha} X^{\beta}\right) \eta^{\mu \nu} .
\end{aligned}
$$


This basis of 14 tensors with four indixes is constructed with two different four-vectors $X^{\mu}$ and $Y^{\mu}$. Note that in order to maintain the symmetries of the effective action each member of the basis has to be symmetric under the interchanges $\mu \leftrightarrow \nu, \alpha \leftrightarrow \beta$ and $(\mu \nu) \leftrightarrow(\alpha \beta)$ [3]. In terms of this tensor basis, the tensor $\mathrm{T}^{\mu \nu, \alpha \beta}(q, k)$, introduced in Sec. IIB, is defined by

$$
\begin{aligned}
\mathrm{T}^{\mu \nu, \alpha \beta}(q, k) \equiv & \mathrm{T}_{3}^{\mu \nu, \alpha \beta}(q, k)+\left\{\frac{1}{4}\left[(k \cdot q)+q^{2}\right]^{2}+\xi k^{2}\left[(k \cdot q)+q^{2}\right]+\xi^{2} k^{4}\right\} \mathrm{T}_{4}^{\mu \nu, \alpha \beta}(q, k) \\
& -\left\{\frac{1}{2}\left[(k \cdot q)+q^{2}\right]+\xi k^{2}\right\} \mathrm{T}_{5}^{\mu \nu, \alpha \beta}(q, k)+\frac{1}{2} \mathrm{~T}_{7}^{\mu \nu, \alpha \beta}(q, k)+\xi \mathrm{T}_{9}^{\mu \nu, \alpha \beta}(q, k) \\
& +\frac{1}{4} \mathrm{~T}_{10}^{\mu \nu, \alpha \beta}(q, k)+\frac{1}{2} \xi \mathrm{T}_{11}^{\mu \nu, \alpha \beta}(q, k)+\xi^{2} \mathrm{~T}_{12}^{\mu \nu, \alpha \beta}(q, k) \\
& -\xi\left\{\frac{1}{2}\left[(k \cdot q)+q^{2}\right]+\xi k^{2}\right\} \mathrm{T}_{13}^{\mu \nu, \alpha \beta}(q, k)-\frac{1}{2}\left\{\frac{1}{2}\left[(k \cdot q)+q^{2}\right]+\xi k^{2}\right\} \mathrm{T}_{14}^{\mu \nu, \alpha \beta}(q, k) .
\end{aligned}
$$

\section{APPENDIX C: SOME COEFFICIENTS AT HIGH TEMPERATURE}

The 14 coefficients $\mathrm{H}_{i}(r)$ needed to write the leading contribution at high temperature of the kernel $\tilde{\mathrm{H}}^{\mu \nu, \alpha \beta}(k)$ in terms of the tensor basis of Appendix B are

$$
\begin{aligned}
& \mathrm{H}_{1}(r)=-\frac{1}{6}-\frac{1}{24} K^{2}-\frac{1}{8} K^{4} L \\
& \mathrm{H}_{2}(r)=-\frac{1}{3}-\frac{1}{12} K^{2}-\frac{5}{24} K^{4}-\frac{5}{8} K^{6} L \\
& \mathrm{H}_{3}(r)=-\frac{1}{3} K^{2}-\frac{7}{12} K^{4}-\frac{35}{24} K^{6}-\frac{35}{8} K^{8} L \\
& \mathrm{H}_{4}(r)=-\frac{1}{24} K^{2}-\frac{1}{8} K^{4} L \\
& \mathrm{H}_{5}(r)=-\frac{1}{12} K^{2}-\frac{5}{24} K^{4}-\frac{5}{8} K^{6} L \\
& \mathrm{H}_{6}(r)=\left(-\frac{1}{12}-\frac{5}{24} K^{2}-\frac{5}{8} K^{4} L\right) r \\
& \mathrm{H}_{7}(r)=\left(-\frac{1}{3}-\frac{7}{12} K^{2}-\frac{35}{24} K^{4}-\frac{35}{8} K^{6} L\right) r \\
& \mathrm{H}_{8}(r)=-\frac{1}{12}+\frac{5}{24} K^{2}-\frac{1}{2} K^{2} L+\frac{5}{8} K^{4} L \\
& \mathrm{H}_{9}(r)=-\frac{1}{6}-\frac{2}{3} K^{2}+\frac{35}{24} K^{4}-\frac{15}{4} K^{4} L+\frac{35}{8} K^{6} L \\
& \mathrm{H}_{10}(r)=-\frac{1}{6}-\frac{2}{3} K^{2}+\frac{35}{24} K^{4}-\frac{15}{4} K^{4} L+\frac{35}{8} K^{6} L \\
& \mathrm{H}_{11}(r)=\left(-\frac{1}{4}+\frac{35}{24} K^{2}-\frac{5}{2} K^{2} L+\frac{35}{8} K^{4} L\right) r \\
& \mathrm{H}_{12}(r)=+\frac{13}{12}-\frac{35}{24} K^{2}-L+5 K^{2} L-\frac{35}{8} K^{4} L \\
& \mathrm{H}_{13}(r)=-\frac{1}{12}+\frac{5}{24} K^{2}-\frac{1}{2} K^{2} L+\frac{5}{8} K^{4} L \\
& \mathrm{H}_{14}(r)=\left(-\frac{1}{12}-\frac{5}{24} K^{2}-\frac{5}{8} K^{4} L\right) r
\end{aligned}
$$

where $K^{\mu} \equiv k^{\mu} /|\vec{k}| \equiv(r, \hat{k})$ and the function $L$ is given by

$$
L(r)=\frac{r}{2} \ln \left(\frac{r+1}{r-1}\right)-1 .
$$


[1] D. J. Gross, M. J. Perry and L. G. Yaffe, Phys. Rev. D 25, 330 (1982).

[2] P. S. Gribosky, J. F. Donoghue and B. R. Holstein, Ann. Phys. (N.Y.) 190, 149 (1989).

[3] A. Rebhan, Nucl. Phys. B351, 706 (1991); U. Kraemmer and A. Rebhan, Phys. Rev. Lett. 67, 793 (1991); A. Rebhan, Nucl. Phys. B368, 479 (1992); H. Nachbagauer, A. K. Rebhan and D. J. Schwarz, Phys. Rev. D 51, R2504 (1995); H. Nachbagauer, A. K. Rebhan and D. J. Schwarz, Phys. Rev. D 53, 882 (1996); H. Nachbagauer, A. K. Rebhan and D. J. Schwarz, Phys. Rev. D 53, 5468 (1996).

[4] A. P. de Almeida, F. T. Brandt and J. Frenkel, Phys. Rev. D 49, 4196 (1994), and references therein; F. T. Brandt and J. Frenkel, The structure of the graviton self-energy at finite temperature, hep-th/9803155

[5] F. T. Brandt, J. Frenkel and J. C. Taylor, Nucl. Phys. B374, 169 (1992); J. Frenkel, E. A. Gaffney and J. C. Taylor, Nucl. Phys. B439, 131 (1995).

[6] R. Jackiw and V. P. Nair, Phys. Rev. D 48, 4991 (1993).

[7] A. Einstein, Ann. Physik 17, 549 (1905); 19, 371 (1906).

[8] H. Nyquist, Phys. Rev. 32, 110 (1928).

[9] H. B. Callen and T. A. Welton, Phys. Rev. 83, 34 (1951); H. B. Callen and R. F. Greene, Phys. Rev. 86, 702 (1952); J. Weber, Phys. Rev. 101, 1620 (1956).

[10] R. Kubo, J. Phys. Soc. Japan 12, 570 (1957).

[11] P. C. Martin and J. Schwinger, Phys. Rev. 115, 1342 (1959); L. P. Kadanoff and P. C. Martin Ann. Phys. (N.Y.) 24, 419 (1963).

[12] W. Bernard and H. B. Callen, Rev. Mod. Phys. 31, 1017 (1959); R. Kubo, Rep. Prog. Phys. 29, 255 (1966); L. Landau, E. Lifshitz and L. Pitaevsky, Statistical Physics, (Pergamon, London, 1980), Vol. 1; R. Kubo, M. Toda and N. Hashitsume, Statistical Physics II, (Springer-Verlag, Berlin, 1985).

[13] J. Schwinger, J. Math. Phys. (N.Y.) 2, 407 (1961); Phys. Rev. 128, 2425 (1962); P. M. Bakshi and K. T. Mahanthappa, J. Math. Phys. (N.Y.) 4, 1 (1963); 4, 12 (1963).

[14] L. V. Keldysh, Zh. Eksp. Teor. Fiz. 47, 1515 (1964) [Sov. Phys. JETP 20, 1018 (1965)].

[15] R. P. Feynman and F. L. Vernon, Ann. Phys. (N.Y.) 24, 118 (1963); R. P. Feynman and A. R. Hibbs, Quantum Mechanics and Path Integrals (McGraw-Hill, New York, 1965).

[16] H. A. Weldon, Phys. Rev. D 26, 1394 (1982).

[17] B.-L. Hu, Phys. Lett. 108B, 19 (1982); 123B, 189 (1983).

[18] A. O. Caldeira and A. J. Leggett, Physica 121A, 587 (1983).

[19] B.-L. Hu, J. P. Paz and Y. Zhang, Phys. Rev. D 45, 2843 (1992); D47, 1576 (1993); B.-L. Hu and A. Matacz, Phys. Rev. D 49, 6612 (1994).

[20] N. D. Birrell and P. C. W. Davies, Quantum Fields in Curved Space, (Cambridge University Press, Cambridge, England, 1982).

[21] B.-L. Hu, Physica A158, 399 (1989).

[22] C.-I. Kuo and L. H. Ford, Phys. Rev. D 47, 4510 (1993).

[23] E. Flanagan and R. M. Wald, Phys. Rev. D 54, 6233 (1996).

[24] N. G. Phillips and B.-L. Hu, Phys. Rev. D 55, 6123 (1997).

[25] E. Calzetta and B.-L. Hu, Phys. Rev. D 49, 6636 (1994).

[26] B.-L. Hu and A. Matacz, Phys. Rev. D 51, 1577 (1995).

[27] B.-L. Hu and S. Sinha, Phys. Rev. D 51, 1587 (1995).

[28] A. Campos and E. Verdaguer, Phys. Rev. D 53, 1927 (1996).

[29] F. C. Lombardo and F. D. Mazzitelli, Phys. Rev. D 55, 3889 (1997).

[30] A. Campos and E. Verdaguer, Int. J. Theor. Phys. 36, 2525 (1997).

[31] E. Calzetta, A. Campos and E. Verdaguer, Phys. Rev. D 56, 2163 (1997).

[32] J. J. Halliwell, Phys. Rev. D 57, 2337 (1998).

[33] R. Martín and E. Verdaguer, in preparation (1998).

[34] K. Chou, Z. Su and B. Hao, Phys. Rev. B 22, 3385 (1980).

[35] K. Chou, Z. Su, B. Hao and L. Yu, Phys. Rep. 118, 1 (1985).

[36] K. Chou, L. Chen, Z. Su and X. Yu, Phys. Rev. B 37, 9810 (1988).

[37] E. Calzetta and B.-L. Hu, Phys. Rev. D 35, 495 (1987).

[38] E. Calzetta and B.-L. Hu, Phys. Rev. D 37, 2878 (1988).

[39] D. Boyanovsky and D-S. Lee, Nucl. Phys. B406, 631 (1993).

[40] D. Boyanovsky, H. J. de Vega and R. Holman, in Second Paris Cosmology Colloquium, Eds. H. J. de Vega and N. Sánchez (World Scientific, Singapure, 1995).

[41] B.-L. Hu, in Proceedings of the Banff/Cap Workshop on Thermal Field Theory, Eds. F. C. Khanna, R. Kobes, G. 
Kunstatter, and H. Umezawa (World Scientific, Singapure, 1994).

[42] A. Campos and E. Verdaguer, Phys. Rev. D 49, 1861 (1994).

[43] M. Gleiser and R. O. Ramos, Phys. Rev. D 50, 2441 (1994).

[44] F. Cooper, S. Habib, Y. Kluger, E. Mottola and J. P. Paz, Phys. Rev. D 50, 2848 (1994).

[45] E. Calzetta and B.-L. Hu, Phys. Rev. D 52, 6770 (1995); E. Calzetta and S. Gonorazky, Phys. Rev. D 55, 1812 (1997); A. Matacz, Phys. Rev. D 55, 1860 (1997); 56, R1836 (1997).

[46] S. A. Ramsey and B.-L. Hu, Phys. Rev. D 56, 678 (1997); S. A. Ramsey, B.-L. Hu and A. Stylianopoulos, Phys. Rev. D $\mathbf{5 7 ,} 6003$ (1998).

[47] B. S. DeWitt, in Quantum Concepts of Space and Time, Eds. R. Penrose and C. J. Isham (Clarendon Press, Oxford, 1986).

[48] R. D. Jordan, Phys. Rev. D 33, 444 (1986); 36, 3593 (1987).

[49] M. Le Bellac, Thermal Field Theory, (Cambridge University Press, Cambridge, England, 1996).

[50] D. S. Jones, The theory of generalized functions, (Cambridge University Press, Cambriedge, England, 1982).

[51] P. Candelas and D. W. Sciama, Phys. Rev. Lett. 38, 1372 (1977).

[52] E. Mottola, Phys. Rev. D 33, 2136 (1986).

[53] R. Mills, Propagators for Many-Particle Systems, (Gordon and Breach, New York, 1969).

[54] G. W. Semenoff and N. Weiss, Phys. Rev. D 31, 689 (1985).

[55] J. P. Paz, Phys. Rev. D 41, 1054 (1990).

[56] A. J. Niemi and G. W. Semenoff, Ann. of Phys. 152, 105 (1984); Nucl. Phys. B230, 181 (1984).

[57] N. P. Landsman and Ch. G. van Weert, Phys. Rep. 145, 141 (1987).

[58] R. L. Kobes and K. L. Kowalski, Phys. Rev. D 34, 513 (1986).

[59] A. Niégawa, Phys. Rev. D 40, 1199 (1989). 